\title{
Chemical abundances of 1111 FGK stars from the HARPS GTO planet search program
}

\author{
II. $\mathrm{Cu}, \mathrm{Zn}, \mathrm{Sr}, \mathrm{Y}, \mathrm{Zr}, \mathrm{Ba}, \mathrm{Ce}, \mathrm{Nd}$, and $\mathrm{Eu}^{\star, \star \star}$ \\ E. Delgado Mena ${ }^{1}$, M. Tsantaki², V. Zh. Adibekyan ${ }^{1}$, S. G. Sousa ${ }^{1,3}$, N. C. Santos ${ }^{1,3}$, \\ J. I. González Hernández ${ }^{4,5}$, and G. Israelian ${ }^{4,5}$

\begin{abstract}
${ }^{1}$ Instituto de Astrofísica e Ciências do Espaço, Universidade do Porto, CAUP, Rua das Estrelas, 4150-762 Porto, Portugal e-mail: Elisa.Delgado@astro.up.pt

2 Instituto de Radioastronomía y Astrofísica, IRyA, UNAM, Campus Morelia, A.P. 3-72, CP 58089 Michoacán, Mexico

3 Departamento de Física e Astronomía, Faculdade de Ciências, Universidade do Porto, 4099-002 Porto, Portugal

4 Instituto de Astrofísica de Canarias, C/ via Lactea, s/n, 38205 La Laguna, Tenerife, Spain

5 Departamento de Astrofísica, Universidad de La Laguna, 38206 La Laguna, Tenerife, Spain
\end{abstract}

Received 31 January 2017 / Accepted 7 May 2017

\begin{abstract}
Aims. To understand the formation and evolution of the different stellar populations within our Galaxy it is essential to combine detailed kinematical and chemical information for large samples of stars. The aim of this work is to explore the chemical abundances of neutron capture elements which are a product of different nucleosynthesis processes taking place in diverse objects in the Galaxy, such as massive stars, asymptotic giant branch (AGB) stars and supernovae (SNe) explosions.

Methods. We derive chemical abundances of $\mathrm{Cu}, \mathrm{Zn}, \mathrm{Sr}, \mathrm{Y}, \mathrm{Zr}, \mathrm{Ba}, \mathrm{Ce}, \mathrm{Nd}$, and $\mathrm{Eu}$ for a large sample of more than $1000 \mathrm{FGK}$ dwarf stars with high-resolution $(R \sim 115000)$ and high-quality spectra from the HARPS-GTO program. The abundances are derived by a standard local thermodynamic equilibrium (LTE) analysis using measured equivalent widths (EWs) injected to the code MOOG and a grid of Kurucz ATLAS9 atmospheres.

Results. We find that thick disc stars are chemically disjunct for $\mathrm{Zn}$ and $\mathrm{Eu}$ and also show on average higher $\mathrm{Zr}$ but lower $\mathrm{Ba}$ and $\mathrm{Y}$ than the thin disc stars. We also discovered that the previously identified high- $\alpha$ metal-rich population is also enhanced in $\mathrm{Cu}, \mathrm{Zn}, \mathrm{Nd}$, and $\mathrm{Eu}$ with respect to the thin disc but presents lower $\mathrm{Ba}$ and $\mathrm{Y}$ abundances on average, following the trend of thick disc stars towards higher metallities and further supporting the different chemical composition of this population. By making a qualitative comparison of $\mathrm{O}$ (pure $\alpha$ ), $\mathrm{Mg}, \mathrm{Eu}$ (pure $r$-process), and $s$-process elements we can distinguish between the contribution of the more massive stars (SNe II for $\alpha$ and $r$-process elements) and the lower mass stars (AGBs) whose contribution to the enrichment of the Galaxy is delayed, due to their longer lifetimes. The ratio of heavy-s to light- $s$ elements of thin disc stars presents the expected behaviour (increasing towards lower metallicities) and can be explained by a major contribution of low-mass AGB stars for $s$-process production at disc metallicities. However, the opposite trend found for thick disc stars suggests that intermediate-mass AGB stars play an important role in the enrichment of the gas from where these stars formed. Previous works in the literature also point to a possible primary production of light- $s$ elements at low metallicities to explain this trend. Finally, we also find an enhancement of light- $s$ elements in the thin disc at super-solar metallicities which could be caused by the contribution of metal-rich AGB stars.

Conclusions. This work proves the utility of homogeneous and high-quality data of modest sample sizes. We find some interesting trends that might help to differentiate thin and thick disc population (such as $[\mathrm{Zn} / \mathrm{Fe}]$ and $[\mathrm{Eu} / \mathrm{Fe}]$ ratios) and that can also provide useful constraints for Galactic chemical evolution models of the different populations in the Galaxy.
\end{abstract}

Key words. stars: abundances - stars: fundamental parameters - Galaxy: evolution - Galaxy: disk - solar neighborhood

\section{Introduction}

In recent years several large spectroscopic surveys have started, such as the Gaia ESO Survey (Gilmore et al. 2012), SEGUE (Yanny et al. 2009), APOGEE (Wilson et al. 2010), RAVE (Steinmetz 2003), and GALAH (Heijmans et al. 2012), helping to improve our vision and understanding of the Galaxy and of

\footnotetext{
* Based on observations collected at the La Silla Observatory, ESO (Chile), with the HARPS spectrograph at the $3.6 \mathrm{~m}$ ESO telescope (ESO runs ID 72.C—0488, 082.C—0212, and 085.C-0063).

$\star \star$ Full Tables 1 and 3 are only available at the CDS via anonymous ftp to cdsarc.u-strasbg. fr $(130.79 .128 .5)$ or via http://cdsarc.u-strasbg.fr/viz-bin/qcat?J/A+A/606/A94
}

the Galactic chemical evolution (GCE). In an interesting work, Lindegren \& Feltzing (2013) show that when the precision is low even very large samples do not allow different stellar populations to be separated. However, very interesting results can be obtained with smaller samples of high-resolution and high $\mathrm{S} / \mathrm{N}$ spectra. This is the case of the high-quality HARPS GTO sample which allowed us to discover a new population of high$\alpha$ metal-rich stars (hereafter homr) never seen before and with different properties than thin disc stars of similar iron metallicity $([\mathrm{Fe} / \mathrm{H}])$ (Adibekyan et al. 2011). The objective of this work is to derive chemical abundances of heavy elements $(Z \geq 29)$ as a continuation of the work started by Adibekyan et al. (2012) for lighter elements using the same sample. Moreover, our 
volume-limited sample contains a significant number of metalrich stars $([\mathrm{Fe} / \mathrm{H}]>0.2 \mathrm{dex})$ which permits the study of the GCE at high metallicities not very often explored in the literature.

The nucleosynthesis of elements heavier than Fe cannot be produced by stellar fusion since it would require energy. Instead, they are created by neutron capture processes. There are two main kinds of neutron capture processes. First, the $s$-process (slow), in which the density of neutrons is low and the timescale between consecutives captures is relatively long. If a recently created nuclei is unstable it will suffer a $\beta$ decay transforming neutrons into protons, hence producing heavier elements along the so-called $s$-process path. Second, the $r$-process (rapid) where the captures take place on very short timescales (shorter than the timescale for $\beta$ decay) and the density of neutrons is high Finally, a marginal contribution of heavy elements is provided by the $p$-process (Burbidge et al. 1957). They are also called $p$ nuclei since they are relatively proton-rich nuclei built by $(p, \gamma)$ and/or $(\gamma, n)$ reactions. Among the elements studied in this work we have $\mathrm{Sr}, \mathrm{Zr}$, and $\mathrm{Y}$, which belong to the first peak of the $s$ process path (also called light- $s$ ), and $\mathrm{Ba}, \mathrm{Ce}$, and $\mathrm{Nd}$, which belong to the second peak (called heavy-s). Europium is an $r$ process element and $\mathrm{Cu}$ and $\mathrm{Zn}$ can be considered special $s$ process elements since their production sites are different to heavier $s$-process elements (see next paragraph). Further information can be obtained in the reviews by e.g. Busso et al. (1999), Sneden et al. (2008), Käppeler et al. (2011).

The bulk composition of $s$-process elements is accounted for by the main- $s$ component, which produces elements with $90 \lesssim A \lesssim 204^{1}$ in the He-intershell of asymptotic giant branch (AGB) stars $\left(1.3 M_{\odot} \lesssim M \lesssim 8 M_{\odot}\right.$ ) (Arlandini et al. 1999; Busso et al. 1999). The ejection of AGB envelopes, leaving the $\mathrm{CO}$ core as a white dwarf, produces the enrichment of the interstellar medium (ISM) with the elements previously processed. On the other hand, elements with $60 \lesssim A \lesssim 90$ are thought to be produced by the weak- $s$ component during He-core and Cshell burning in massive stars with $M \gtrsim 8 M_{\odot}$. Part of the material processed inside these massive stars is not altered by the explosive nucleosynthesis of $\mathrm{SNe}$ II and is ejected to enrich the ISM (e.g. Kappeler et al. 1989; Prantzos et al. 1990; Raiteri et al. 1993). A third component, the strong- $s$, provides the stable isotopes at the termination point of the $s$-path, ${ }^{208} \mathrm{~Pb}$ and ${ }^{209} \mathrm{Bi}$ (e.g. Bisterzo et al. 2016). Finally, the production sites for $r$-process elements are still debated, but they are presumably associated with explosive conditions in supernovae, due to the extreme neutron density needed (e.g. Cowan \& Thielemann 2004; Sneden et al. 2008, and refererences therein).

As we have seen, the $s$-process produces the bulk composition of most of the elements analysed in this work. However, the $r$-process contribution is non-negligible in several cases. For example, $s$-process contribution at the time of the solar system formation for the elements of the first peak is $85 \%$ for $\mathrm{Sr}, 92 \%$ for Y, and $83 \%$ for Zr. However, the heavy-s have a smaller contribution from the $s$-process: $81 \%$ for $\mathrm{Ba}, 77 \%$ for Ce, and $56 \%$ for $\mathrm{Nd}$. Finally, Eu is considered a pure- $r$-process element since its $s$-process contribution is only 7\% (Arlandini et al. 1999). A recent study by Bisterzo et al. (2016) gives updated percentages of $s$-process contribution for $\mathrm{Sr}(67 \%), \mathrm{Y}(70 \%), \mathrm{Zr}(64 \%), \mathrm{Ba}$ $(83 \%)$, and $\mathrm{Ce}(81 \%)$.

By studying different populations of stars at different metallicities we can understand which processes played a major role in the production of such elements at a given moment of the evolution of the Galaxy, providing constraints for the current

\footnotetext{
$A$ is the atomic mass number.
}

models of GCE. This is the main objective of this paper, which is organized as follows. Section 2 briefly describes the collected data together with the determination of stellar parameters. In Sect. 3 we detail the derivation of abundances and the error treatment. In Sect. 4 we discuss the behaviour of different abundance ratios for the population of thin disc, thick disc, and homr stars. Finally, we present our conclusions in Sect. 5.

\section{Observations and stellar parameters}

The baseline sample used in this work is formed by 1111 FGK stars observed within the context of the HARPS GTO programs. It is a combination of three HARPS subsamples hereafter called HARPS-1 (Mayor et al. 2003), HARPS-2 (Lo Curto et al. 2010), and HARPS-4 (Santos et al. 2011). The individual spectra of each star were reduced using the HARPS pipeline and then combined with IRAF ${ }^{2}$ after correcting for its radial velocity shift. The final spectra have a resolution of $R \sim 115000$ and high signal-to-noise ratios $(55 \%$ of the spectra have a $\mathrm{S} / \mathrm{N}$ higher than 200). The total sample is composed of 136 stars with planets and 975 stars without detected planets. Chemical abundances of these samples for refractory elements with $A<29$ can be found in Adibekyan et al. (2012) together with oxygen (Bertran de Lis et al. 2015), carbon (Suárez-Andrés et al. 2017), lithium (Delgado Mena et al. 2014, 2015), and nitrogen abundances (Suárez-Andrés et al. 2016, only for a small fraction of stars).

The stellar parameters, namely the effective temperature $\left(T_{\text {eff }}\right)$, surface gravity $(\log g)$, metallicity $([\mathrm{Fe} / \mathrm{H}])$, and microturbulence $\left(\xi_{t}\right)$, were taken from Sousa et al. (2008, 2011a,b). All atmospheric parameters were determined in a homogeneous way based on the measurements of the equivalent widths (EW) of $\mathrm{Fe} \mathrm{I}$ and Fe II lines, and on iron excitation and ionization equilibrium. The effective temperatures of cool stars derived with the linelist of Sousa et al. (2008) were overestimated compared to the infrared flux method. Therefore, in Tsantaki et al. (2013) we compile a reduced linelist specially selected to eliminate lines that suffer from blending effects which are strongly present for the cooler stars and have a significant effect mainly on the determination of temperature. We used this linelist in the complete sample to re-derive the parameters of stars cooler than $5200 \mathrm{~K}$ since only the stars in the subsample HARPS-1 were corrected in Tsantaki et al. (2013). In Fig. 1 we present the comparison between the previous parameters and the parameters of this work for the 297 stars with $T_{\text {eff }}<5200 \mathrm{~K}$. The new $T_{\text {eff }}$ are corrected to lower values, with an average difference of $-95 \pm 56 \mathrm{~K}$, whereas $\log g$ is not affected sigificantly (the mean difference is $-0.09 \pm 0.09 \mathrm{dex})$ and even less for $[\mathrm{Fe} / \mathrm{H}]$ (the average difference is $0.005 \pm 0.03 \mathrm{dex}$ ). The remaining stars (above $5200 \mathrm{~K}$ ) kept their initial parameters. For some of these cool stars the derivation of parameters with the new linelist did not converge and thus the final sample is composed of 1059 stars. The estimation of errors in parameters is done in the same way as in (Tsantaki et al. 2013).

Additionally, we calculated the trigonometric $\log g$ (see Eq. (1) from Santos et al. 2004) using the new HIPPARCos parallaxes (van Leeuwen 2007), $V$ magnitudes from Simbad, bolometric corrections based on Flower (1996) and

2 IRAF is distributed by National Optical Astronomy Observatories, operated by the Association of Universities for Research in Astronomy, Inc., under contract with the National Science Foundation, USA 

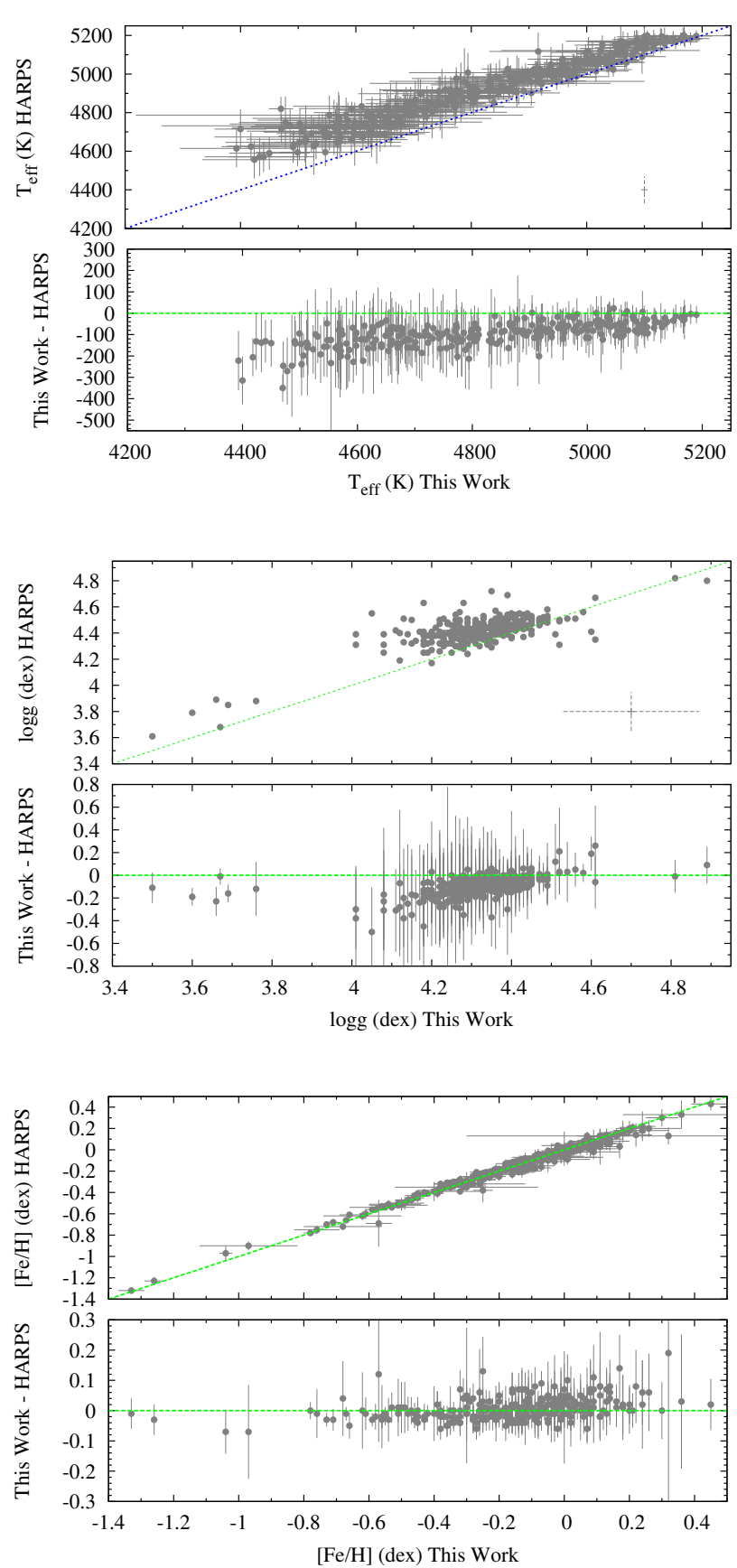

Fig. 1. Comparison between the temperature derived with the cool line list of this work with the previous parameters (upper panel). Same for $\log g$ (middle panel) and [Fe/H] (bottom panel).

Torres (2010), solar magnitudes from (Bessell et al. 1998), stellar masses, and spectroscopic $T_{\text {eff }}$. Stellar masses are derived from the PARAM v1.3 tool $^{3}$ using the PARSEC theoretical isochrones from (Bressan et al. 2012) and a Bayesian estimation method (da Silva et al. 2006). The stellar masses are obtained using the observational information $(V$ mag teff, $[\mathrm{Fe} / \mathrm{H}]$, and parallax) to compute the probability density functions of the main stellar properties (mass, luminosity, and age). The Bayesian inference is applied taking into account priors for the initial mass function Chabrier (2001) and a constant star formation rate (SFR). No correction for interstellar reddening is needed since all stars are at a short distance from us.

\footnotetext{
3 http://stev.oapd.inaf.it/cgi-bin/param
}

The correlation of $\log g_{\text {spec }}-\log g_{\mathrm{HIP}}$ with $T_{\text {eff }}$ has already been reported in some studies, but no clear explanation has been proposed for why the ionization balance is not properly handled (e.g. Tsantaki et al. 2013; Bensby et al. 2014). Interestingly, the differences between $\log g_{\text {spec }}$ and $\log g$ derived from other more model-independent methods, such as the transit fit of planet hosts and asteroseismic analysis, show a similar correlation with $T_{\text {eff }}$ (Mortier et al. 2014). Moreover, it has been shown that $\log g$ derived from the ionization balance does not follow isochrones in the Hertzsprung-Russell (HR) diagram, opposite to what happens to trigonometric $\log g$ (see lower panel of Fig. 2). Therefore, we decided to derive a correction of our spectroscopic $\log g$ to have more realistic values. The comparison between the trigonometric gravities and the spectroscopic values is presented in the upper panel of Fig. 2. We calculated the correction to the spectroscopic values using linear fits in three different $T_{\text {eff }}$ ranges corresponding to different spectral types and only for stars with parallax errors smaller than $5 \%$ (67\% of our sample). A cut in the parallax error is necessary because biases in our distance estimations are introduced by simply inverting the parallax (e.g. Astraatmadja \& Bailer-Jones 2016). The average differences between the spectrocopic $\log g$ and the trigonometric $\log g$ are $-0.22 \pm 0.13,0.02 \pm 0.12$, and $0.21 \pm 0.13$ dex for the cool solar temperature and hot stars, respectively.

The corrections are presented in Eqs. (1)-(3) and are suggested in order to correct the $\log g$ values derived from this method. With the new parallax releases of the Gaia mission, we will obtain very precise trigonometric gravities for millions of stars that will help us improve our spectroscopic gravities from calibrations such as those presented in this work. In the HR diagram of Fig. 2 we can see how the new $\log g$ corrected values follow the isochrones better. Thus, for the derivation of abundances explained in next section we used the following corrected $\log g$ :

$$
\begin{gathered}
\log g_{\mathrm{cor}}=\log g_{\mathrm{spec}}-3.364 \times 10^{-4} T_{\mathrm{eff}}+1.843 \\
\quad\left(\text { for } T_{\mathrm{eff}}<5200 \mathrm{~K}\right) \\
\log g_{\mathrm{cor}}=\log g_{\text {spec }}-2.521 \times 10^{-4} T_{\mathrm{eff}}+1.416 \\
\left(\text { for } 5200 \mathrm{~K} \leq T_{\mathrm{eff}} \leq 6100 \mathrm{~K}\right) \\
\log g_{\mathrm{cor}}=\log g_{\mathrm{spec}}-4.217 \times 10^{-4} T_{\mathrm{eff}}+2.455
\end{gathered}
$$$$
\text { (for } T_{\mathrm{eff}}>6100 \mathrm{~K} \text { ). }
$$

The errors for the corrected $\log g$ are the same as for the spectroscopic values; the difference is less than 0.01 dex. The complete table with the updated parameters is available at the CDS; a sample of our results is shown in Table 1.

\section{Derivation of chemical abundances}

The chemical abundances for most of the elements were derived under a standard LTE analysis with the 2014 version of the code MOOG (Sneden 1973) using the abfind driver. For the lines affected by hyperfine splitting (HFS) we used the blends driver. A grid of Kurucz ATLAS9 atmospheres (Kurucz 1993) were used as input along with the equivalent widths (EWs) and the atomic parameters, wavelength $(\lambda)$, excitation energy of the lower energy level $(\chi)$, and oscillator strength $(\log g f)$ of each line. The EWs of the different lines were measured automatically with version 2 of the ARES program ${ }^{4}$ (Sousa et al. 2015, 2007). The input parameters for ARES were adjusted for the different atomic lines used in this study. We measured the EWs of ten stars with

\footnotetext{
4 The ARES code can be downloaded at http://www . astro.up.pt/ sousasag/ares/
} 
Table 1. Sample table of the derived stellar parameters for each star including the spectroscopic, trigonometric and corrected log $g$.

\begin{tabular}{lcccccc}
\hline \hline Star & $\begin{array}{c}T_{\text {eff }} \\
(\mathrm{K})\end{array}$ & $\begin{array}{c}\log g_{\text {spec }} \\
\left(\mathrm{cm} \mathrm{s}^{-2}\right)\end{array}$ & $\begin{array}{c}\log g_{\mathrm{HIP}} \\
\left(\mathrm{cm} \mathrm{s}^{-2}\right)\end{array}$ & {$[\mathrm{Fe} / \mathrm{H}]$} & $\begin{array}{c}\xi_{t} \\
\left(\mathrm{~km} \mathrm{~s}^{-1}\right)\end{array}$ & $\begin{array}{c}\log g_{\text {cor }} \\
\left(\mathrm{cm} \mathrm{s}^{-2}\right)\end{array}$ \\
\hline HD 144411 & $4839 \pm 72.0$ & $4.45 \pm 0.14$ & $4.60 \pm 0.08$ & $-0.32 \pm 0.02$ & $0.23 \pm 0.38$ & 4.67 \\
HIP 33392 & $4843 \pm 70.4$ & $4.39 \pm 0.17$ & $4.56 \pm 0.08$ & $-0.05 \pm 0.04$ & $0.49 \pm 0.19$ & 4.60 \\
HD 154577 & $4847 \pm 35.0$ & $4.48 \pm 0.07$ & $4.58 \pm 0.02$ & $-0.73 \pm 0.01$ & $0.31 \pm 0.19$ & 4.69 \\
HIP 32812 & $4849 \pm 72.4$ & $4.27 \pm 0.18$ & $4.55 \pm 0.09$ & $-0.01 \pm 0.06$ & $0.20 \pm 0.56$ & 4.48 \\
HD 2025 & $4851 \pm 49.0$ & $4.49 \pm 0.13$ & $4.58 \pm 0.03$ & $-0.37 \pm 0.02$ & $0.51 \pm 0.18$ & 4.70 \\
$\ldots$ & $\ldots$ & $\ldots$ & $\ldots$ & $\ldots$ & $\ldots$ & $\ldots$ \\
\hline
\end{tabular}

Notes. The full table is available at the CDS.
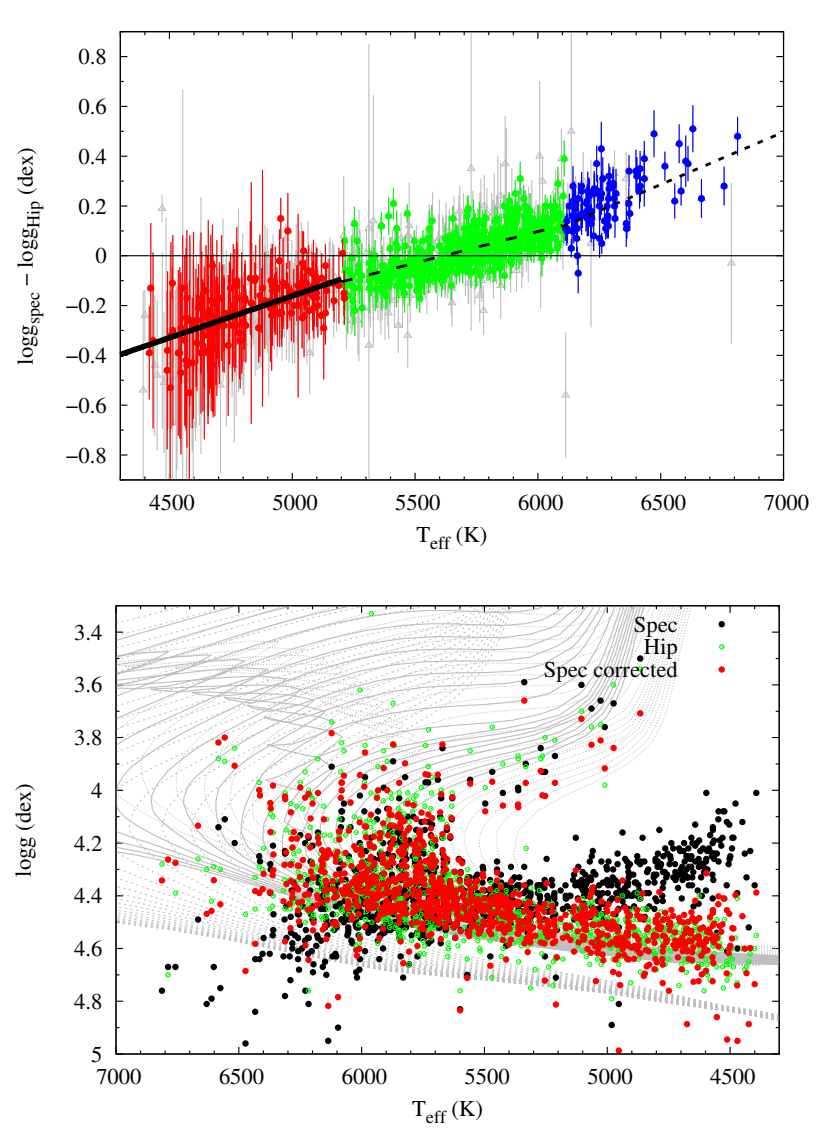

Fig. 2. Upper panel: comparison between the $\log g$ from spectroscopy and that derived from HIPPARCos parallaxes as a function of $T_{\text {eff }}$. The different lines represent linear fits in three different $T_{\text {eff }}$ bins. The stars with error in parallax higher than 5\% (not considered for the fit) are represented by grey triangles. Lower panel: HR diagram with our spectroscopic $\log g$, our corrected $\log g$ values, and the $\log g$ using the HIPPARCos parallaxes.

different $T_{\text {eff }}$ and $[\mathrm{Fe} / \mathrm{H}]$ manually, with the task splot in IRAF, and compared them with the obtained values by ARES using different input parameters until we found the best approach for each line. We also measured the EWs in a solar reflected light spectrum of the Vesta asteroid (obtained by combining several high S/N spectra observed with HARPS) in order to derive our reference abundances. The atomic data, EWs, and derived abundances for the Sun are shown in Table 2. If not specified in following subsections, the atomic parameters of the lines and the Van der Waals damping constants, $\log \left(\gamma_{6} / N_{\mathrm{H}}\right)$, were retrieved from the VALD3 database ${ }^{5}$ (Ryabchikova et al. 2015). When no

\footnotetext{
5 http://vald.astro.univie.ac.at/ vald3/php/vald.php
}

damping constants were available in VALD3, the Unsöld approximation with an enhancement factor was considered. This enhancement, $E$, was calculated as recommended by the Blackwell group ( $E=1+0.67 \chi_{1}$, damping option 2 within MOOG). We did not attempt to calibrate the $\log g f$ values with standard solar abundances; therefore, we derived the $[\mathrm{X} / \mathrm{Fe}]$ ratios line by line with respect to the solar abundances shown in Table 2 to later combine them as explained in next subsections. All the abundances are provided in the electronic tables; a portion of our results is shown in Table 3.

\subsection{Error determination}

In order to determine the uncertainties on the abundances we have to consider the errors on the EW measurements, the errors on the atomic parameters of the lines, and the errors due to the uncertainties in the atmospheric parameters. We consider the line-to-line scatter as an approximation of the error on the measurement of EW (uncertainties in continuum position, blends, etc.) and the errors on atomic parameters. On the other hand, the abundance uncertainties due to the errors on stellar parameters were estimated by calculating the abundance differences when one of each of the stellar parameters was modified by its individual error. The average abundance sensitivity for each element is shown in Table 4 for the same three groups of stars depending on $T_{\text {eff }}$ as was done in Adibekyan et al. (2012): "low $T_{\text {eff " }}$ stars with $T_{\text {eff }}<5277 \mathrm{~K}$; "solar" stars with $T_{\text {eff }}=T_{\odot} \pm 500 \mathrm{~K}$; and "high Teff" stars with $T_{\text {eff }}>6277 \mathrm{~K}$. The average errors on $T_{\text {eff }}$ are 64,24 , and $46 \mathrm{~K}$ for cool, Sun-like, and hot star groups, respectively. The average errors in $\log g$ are $0.17,0.03$, and $0.05 \mathrm{dex}$; in $\xi_{\mathrm{t}}$ are $0.33,0.04$, and $0.08 \mathrm{~km} \mathrm{~s}^{-1}$; and in $[\mathrm{Fe} / \mathrm{H}]$ are $0.04,0.02$, 0.03 dex for the three groups, respectively. We can see that for solar $T_{\text {eff }}$ and hotter stars the dominant error is due to the lineto-line scatter. However, for cool stars the errors on the stellar parameters are larger and thus they translate into significant errors on abundances, especially those related with the $\log g$ and $\xi_{\mathrm{t}}$ uncertainties. The neutral species are more sensitive to errors in $T_{\text {eff }}$, while ionized species suffer a higher variation due to changes in $\log g$. Finally, the errors on microturbulence mainly affect the abundances of species with strong lines such as $\mathrm{Sr}$, $\mathrm{Y}$, and $\mathrm{Ba}$, which are more important for cool stars. The final abundance errors are given by the quadratic sum of all these individual errors.

\subsection{Cu abundances}

Copper abundances are based on four neutral lines for which we consider HFS. The atomic parameters and isotopic ratios for 

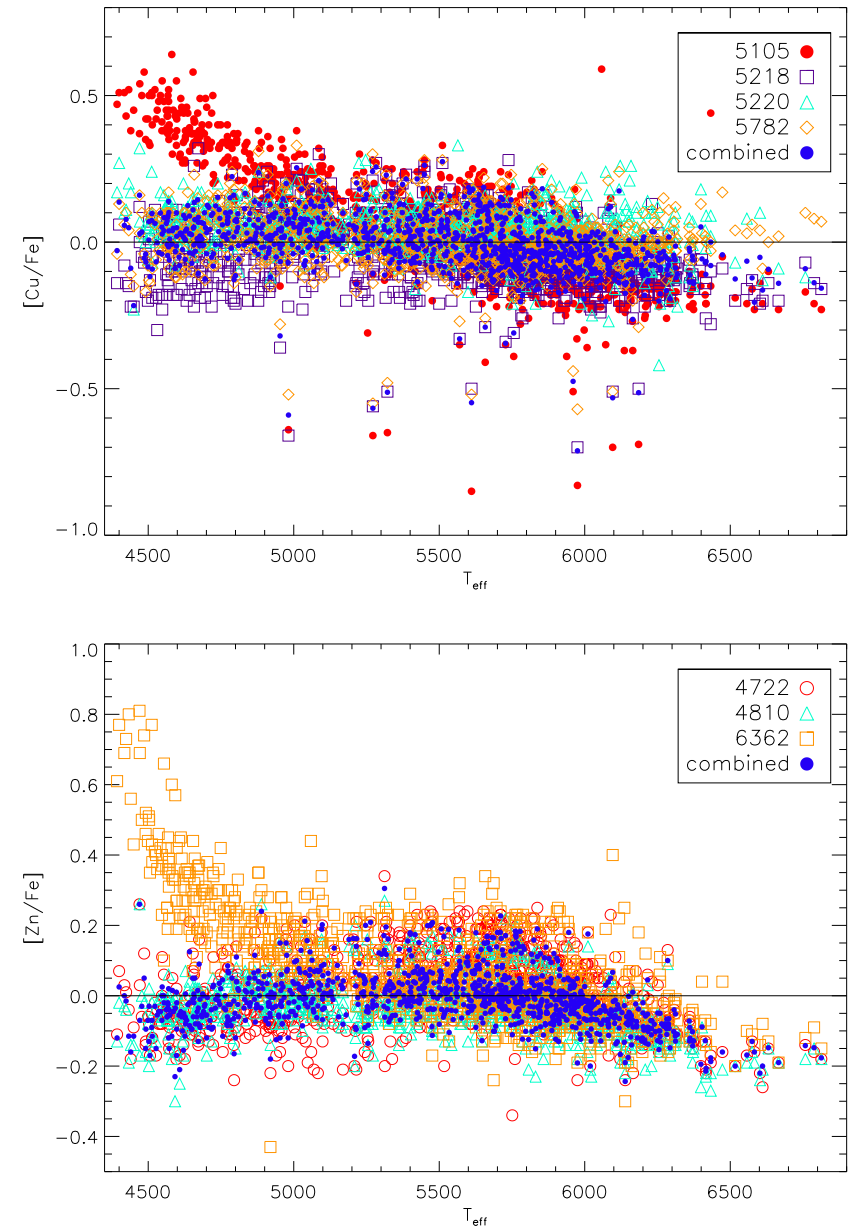

Fig. 3. $[\mathrm{Cu} / \mathrm{Fe}]$ and $[\mathrm{Zn} / \mathrm{Fe}]$ for each line as a function of $T_{\text {eff }}$ together with the final adopted abundances for each star (blue filled circles).

those lines were taken from the Kurucz database ${ }^{6}$. The lines at $5105 \AA$ and $5782 \AA$ are the most affected by HFS. The former becomes very strong at low temperatures, probably due to an unknown blend, thus the abundances are also very high, as can be seen in Fig. 3. In the Sun we also obtain the highest $\mathrm{Cu}$ abundance with this line (see Table 2). Therefore, we decided to discard this line for stars cooler than $5200 \mathrm{~K}$ based on visual inspection of the abundances. The line at $5218 \AA$ gets blended for cool stars and we had to measure it manually in several stars because ARES was not able to deblend it in a correct way. Furthermore, we also inspected visually the measurement of $\mathrm{Cu}$ lines in our most metal-poor stars and in some cases we had to measure with IRAF some weak lines that ARES could not fit. We discard the line at $5220 \AA$ for metal-poor stars because it becomes very weak $(E W<5 \mathrm{~mA})$. To obtain the final abundances we calculated the weighted mean (WM) whereby we consider the distance from the median abundance as a weight. As described in Adibekyan et al. (2015) this method is a good approach that can be used without removing suspected outlier lines.

\subsection{Zn abundances}

Zinc abundances are determined with three neutral lines (see Fig. 3). The line at $6362 \AA$ is located in a depressed continuum region due to a $\mathrm{Ca}$ I auto-ionization line (e.g. Barbuy et al. 2015, and references therein) and for some stars ARES cannot measure

\footnotetext{
6 http://kurucz .harvard .edu/linelists/gfhyperall/
}

Table 2. Atomic parameters for the lines used in this work together with EWs and absolute abundances in the Sun from our Vesta combined spectrum.

\begin{tabular}{|c|c|c|c|c|c|}
\hline Element & $\lambda(\AA)$ & $\chi_{1}(\mathrm{eV})$ & $\log g f$ & $E W(\mathrm{~m} \AA)$ & $\log (A)$ \\
\hline $\mathrm{Cu} \mathrm{I}^{*}$ & 5105.55 & 1.39 & -1.516 & 93.0 & 4.245 \\
\hline $\mathrm{Cu} \mathrm{I}^{*}$ & 5218.21 & 3.82 & 0.476 & 53.5 & 4.088 \\
\hline $\mathrm{Cu} \mathrm{I}^{*}$ & 5220.09 & 3.82 & -0.448 & 16.2 & 4.088 \\
\hline $\mathrm{Cu} \mathrm{I}^{*}$ & 5782.12 & 1.64 & -1.720 & 79.9 & 4.093 \\
\hline $\mathrm{Zn} \mathrm{I}$ & 4722.16 & 4.03 & -0.338 & 67.1 & 4.553 \\
\hline $\mathrm{Zn} \mathrm{I}$ & 4810.54 & 4.08 & -0.137 & 74.0 & 4.530 \\
\hline Zn I & 6362.35 & 5.80 & 0.150 & 20.4 & 4.506 \\
\hline Sr I & 4607.34 & 0.00 & 0.283 & 47.2 & 2.780 \\
\hline Y II & 4374.94 & 0.41 & 0.160 & 86.2 & 2.318 \\
\hline Y II & 4398.01 & 0.13 & -1.000 & 46.5 & 2.097 \\
\hline $\mathrm{Y} \mathrm{II}^{a}$ & 4854.87 & 0.99 & -0.380 & 48.8 & 2.326 \\
\hline Y II & 4900.12 & 1.03 & -0.090 & 55.7 & 2.258 \\
\hline Y II & 5087.43 & 1.08 & -0.170 & 48.5 & 2.178 \\
\hline Y II & 5200.42 & 0.99 & -0.570 & 37.5 & 2.189 \\
\hline Y II & 5402.78 & 1.84 & -0.630 & 11.7 & 2.273 \\
\hline Zr I & 4805.87 & 0.69 & -0.420 & 1.7 & 2.596 \\
\hline $\mathrm{ZrI}$ & 4815.63 & 0.60 & -0.030 & 3.0 & 2.378 \\
\hline $\mathrm{ZrI}$ & 6127.44 & 0.15 & -1.060 & 2.7 & 2.818 \\
\hline $\mathrm{ZrI}$ & 6134.55 & 0.00 & -1.280 & 2.8 & 2.899 \\
\hline $\mathrm{ZrI}$ & 6143.20 & 0.07 & -1.100 & 3.0 & 2.820 \\
\hline Zr II & 4050.32 & 0.71 & -1.060 & 23.3 & 2.604 \\
\hline Zr II & 4208.98 & 0.71 & -0.510 & 43.7 & 2.622 \\
\hline Zr II & 4379.74 & 1.53 & -0.356 & 28.7 & 2.809 \\
\hline Zr II & 5112.28 & 1.67 & -0.850 & 9.3 & 2.697 \\
\hline $\mathrm{Ba} \mathrm{II}^{*}$ & 5853.69 & 0.60 & -1.010 & 64.0 & 2.298 \\
\hline Ba II* & 6141.73 & 0.70 & -0.070 & 112.7 & 2.256 \\
\hline Ba II* & 6496.91 & 0.60 & -0.377 & 97.4 & 2.210 \\
\hline Ce II & 4523.08 & 0.52 & 0.040 & 15.3 & 1.630 \\
\hline Ce II & 4628.16 & 0.52 & 0.230 & 20.4 & 1.609 \\
\hline Ce II & 4773.96 & 0.92 & 0.250 & 10.8 & 1.603 \\
\hline Ce II & 5274.23 & 1.04 & 0.130 & 8.7 & 1.696 \\
\hline Nd II* & 4811.34 & 0.06 & -1.140 & 10.3 & 1.928 \\
\hline Nd II* & 4989.95 & 0.63 & -0.500 & 7.9 & 1.700 \\
\hline Nd II* & 5092.80 & 0.38 & -0.610 & 8.0 & 1.566 \\
\hline Nd II* & 5130.59 & 1.30 & 0.450 & 14.6 & 1.739 \\
\hline Eu II* & 6645.13 & 1.38 & -0.200 & 5.8 & 0.670 \\
\hline
\end{tabular}

Notes. ${ }^{(*)}$ Lines for which HFS is considered. ${ }^{(a)}$ Discarded line.

it correctly, thus we remeasure this line when the abundance is very different to the other lines. Moreover, for cool stars this line becomes weaker and blended with a nearby feature, thus giving higher than average abundances. Therefore, we decided to discard this line for stars cooler than $5000 \mathrm{~K}$ so the final abundance for these stars is the average of the abundance given by the other two lines. For hotter stars we derived the WM as final abundance. In order to check that the line at $6362 \AA$ is not affecting our final results we compared our final abundances with the abundance obtained using only the first two lines and the results are very similar except for a few of the most metal-rich stars where the abundances can increase up to $\sim 0.1$ dex. Since this difference 
Table 3. Sample table of the derived abundances of the elements, error, and number of measured lines for each star.

\begin{tabular}{|c|c|c|c|c|c|c|c|c|c|c|c|c|}
\hline Star & $S / N$ & Classif. & {$[\mathrm{Cu} / \mathrm{Fe}]$} & $\sigma_{\mathrm{Cu}}$ & $n_{\mathrm{Cu}}$ & {$[\mathrm{Zn} / \mathrm{Fe}]$} & $\sigma_{Z n}$ & $n_{\mathrm{Zn}}$ & {$[\mathrm{Sr} / \mathrm{Fe}]$} & $\sigma_{\mathrm{Sr}}$ & $n_{\mathrm{Sr}}$ & $\ldots$ \\
\hline HD 181720 & 695 & thick & -0.039 & 0.056 & 4 & 0.131 & 0.023 & 3 & -0.071 & 0.023 & 1 & \\
\hline HD 131218 & 61 & thin & -0.131 & 0.028 & 4 & -0.101 & 0.020 & 3 & 0.054 & 0.146 & 1 & $\ldots$ \\
\hline HD 111031 & 987 & thin & 0.101 & 0.059 & 4 & 0.009 & 0.059 & 3 & -0.044 & 0.027 & 1 & $\ldots$ \\
\hline HD 183658 & 370 & thin & 0.005 & 0.017 & 4 & -0.003 & 0.034 & 3 & -0.030 & 0.029 & 1 & $\ldots$ \\
\hline HD 107148 & 110 & thin & 0.102 & 0.029 & 4 & -0.007 & 0.027 & 3 & -0.014 & 0.092 & 1 & $\ldots$ \\
\hline$\ldots$ & $\ldots$ & $\ldots$ & $\ldots$ & $\ldots$ & $\ldots$ & $\ldots \ldots$ & $\ldots$ & $\ldots$ & $\ldots$ & $\ldots$ & $\ldots$ & ... \\
\hline
\end{tabular}

Notes. The full table is available at the CDS.

affects a very small percentage of our sample we continued to use it for $T_{\text {eff }}>5000 \mathrm{~K}$.

\subsection{Sr abundances}

To derive $\mathrm{Sr}$ abundances we only used one strong neutral line at $4607 \AA$. Thus, we cannot estimate the error due to the uncertainty in the continuum placement using the line-to-line scatter. For this case we calculated the errors on EWs following Cayrel (1988) by using the FWHM of the lines provided by ARES. The calculated uncertainty takes into account the statistical photometric error due to the noise in each pixel and the error related to the continuum placement, which is the dominant contribution to the error (Cayrel 1988; Bertran de Lis et al. 2015). These errors are then propagated to derive the abundance uncertainties for each line. We show the $[\mathrm{Sr} / \mathrm{Fe}]$ ratios as a function of $T_{\text {eff }}$ in Fig. 8.

\section{5. $Y$ abundances}

We analysed the $\mathrm{Y}$ abundances for seven Y II lines. We found that the line at $4854 \AA$ shows a strong trend of growing abundances for lower $T_{\text {eff }}$ and decreasing abundances for hotter stars, thus we discarded that line. The final abundances are derived as the WM of the six remaining lines. In Fig. 5 we show the discarded line together with only three out of the six final lines for clarity.

\section{6. $\mathrm{Zr}$ abundances}

Zirconium abundances are based on four $\mathrm{Zr}$ II lines. The lines at $4208 \AA$ and $5112 \AA$ become very blended for stars cooler than $5300 \mathrm{~K}$ and ARES cannot separate them; therefore, we used only these lines for hotter stars (see Fig. 4). The line at $4379 \AA$ also seems to give overestimated abundances for cooler stars. Therefore, for stars cooler than $5300 \mathrm{~K}$ we only considered the line at $4050 \AA$ because it does not show a strong trend with $T_{\text {eff }}$ as happens for the other three lines. For hotter stars the final abundances are calculated using the WM of the four lines. In order to improve our abundances for cool stars we searched for reliable $\mathrm{Zr}$ I lines since they are stronger for these stars. However, these lines have EWs smaller than $10 \mathrm{~m} \AA$ for stars above $\sim 5500 \mathrm{~K}$, thus $\mathrm{ZrI}$ abundances for hotter stars must be considered with caution. Indeed, those lines are below $3 \mathrm{~m} \AA$ for the Sun and even with our high-quality solar spectra the errors are non-negligible. On the other hand, for cool stars the agreement between the absolute abundances among the $\mathrm{ZrI}$ lines is much better than the line-by-line differential values with respect to the Sun because the solar absolute abundances of Zr I show a great scatter (see
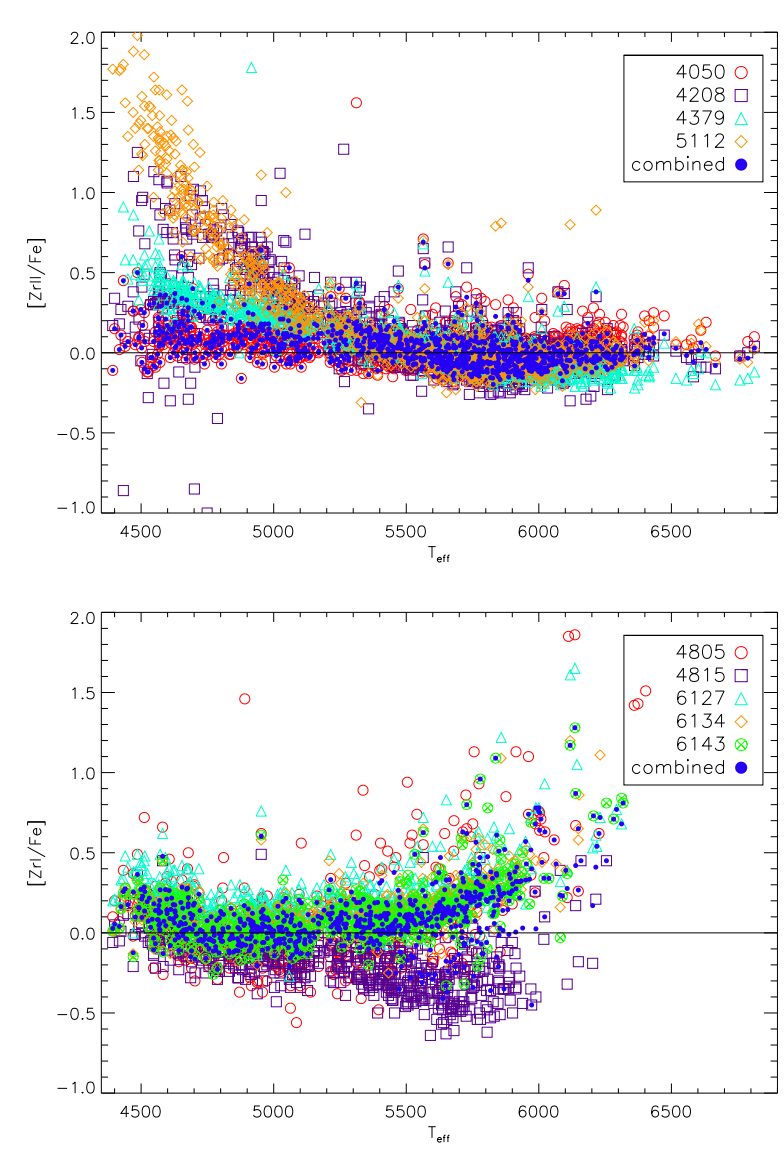

Fig. 4. $[\mathrm{ZrII} / \mathrm{Fe}]$ and $[\mathrm{ZrI} / \mathrm{Fe}]$ for each line as a function of $T_{\text {eff }}$ together with the final abundance adopted for each star (blue filled circles).

Table 2). For cool stars, the absolute abundances of Zr I lines are similar to the abundances of Zr II-4050 $\AA$. Therefore, to calculate $[\mathrm{Zr} \mathrm{I} / \mathrm{Fe}]$ we derived the $\mathrm{WM}$ of the absolute abundances and then subtracted the solar value for $\mathrm{Zr}$ II-4050 A, i.e. 2.60 dex.

\subsection{Ba abundances}

Barium abundances are derived by measuring three strong Ba II lines for which we consider HFS splitting. The atomic parameters and isotopic ratios for those lines were taken from Prochaska et al. (2000). The three lines do not show a strong trend with $T_{\text {eff }}$ (see Fig. 5) thus the final abundances are derived as their WM. Previous works have warned about the possible NLTE effects on $\mathrm{Ba}$ abundances of hot stars (e.g. Bensby et al. 2014). In Fig. 6 we show that although $\mathrm{Ba}$ abundances for stars with $T_{\text {eff }}>6100 \mathrm{~K}$ are higher on average around solar metallicity, they are well mixed among the 
E. Delgado Mena et al.: Chemical abundances of 1111 FGK stars from the HARPS GTO planet search program

Table 4. Average abundance sensitivities of the studied elements to changes of each parameter by their individual $\sigma$.

\begin{tabular}{|c|c|c|c|c|c|c|c|c|c|c|}
\hline & $\mathrm{Cu} \mathrm{I}$ & Zn I & Sr I & Y II & $\mathrm{ZrI}$ & Zr II & $\mathrm{Ba} \mathrm{II}$ & Ce II & Nd II & Eu II \\
\hline \multicolumn{11}{|c|}{ Line-to-line scatter/continuum error } \\
\hline Low $T_{\text {eff }}$ & \pm 0.04 & \pm 0.04 & \pm 0.04 & \pm 0.08 & \pm 0.07 & \pm 0.07 & \pm 0.04 & \pm 0.06 & \pm 0.06 & \pm 0.10 \\
\hline Solar & \pm 0.04 & \pm 0.03 & \pm 0.05 & \pm 0.04 & - & \pm 0.04 & \pm 0.02 & \pm 0.05 & \pm 0.04 & \pm 0.09 \\
\hline High $T_{\text {eff }}$ & \pm 0.07 & \pm 0.03 & \pm 0.06 & \pm 0.06 & - & \pm 0.07 & \pm 0.03 & \pm 0.05 & \pm 0.06 & \pm 0.12 \\
\hline \multicolumn{11}{|c|}{$\Delta T_{\mathrm{eff}}= \pm \sigma_{T_{\mathrm{eff}}}$} \\
\hline Low $T_{\text {eff }}$ & \pm 0.01 & $\mp 0.02$ & \pm 0.10 & \pm 0.01 & \pm 0.10 & \pm 0.01 & \pm 0.02 & \pm 0.02 & \pm 0.02 & $\mp 0.00$ \\
\hline Solar & \pm 0.02 & \pm 0.00 & \pm 0.03 & \pm 0.00 & - & \pm 0.00 & \pm 0.01 & \pm 0.01 & \pm 0.01 & \pm 0.00 \\
\hline High $T_{\text {eff }}$ & \pm 0.03 & \pm 0.02 & \pm 0.04 & \pm 0.02 & - & \pm 0.01 & \pm 0.02 & \pm 0.02 & \pm 0.02 & \pm 0.01 \\
\hline \multicolumn{11}{|c|}{$\Delta[\mathrm{Fe} / \mathrm{H}]= \pm \sigma_{[F e / H]}$} \\
\hline Low $T_{\text {eff }}$ & \pm 0.01 & \pm 0.02 & \pm 0.01 & \pm 0.02 & \pm 0.00 & \pm 0.02 & \pm 0.02 & \pm 0.02 & \pm 0.01 & \pm 0.01 \\
\hline Solar & \pm 0.00 & \pm 0.00 & \pm 0.00 & \pm 0.01 & - & \pm 0.00 & \pm 0.01 & \pm 0.01 & \pm 0.01 & \pm 0.00 \\
\hline High $T_{\text {eff }}$ & \pm 0.00 & \pm 0.00 & \pm 0.00 & \pm 0.01 & - & \pm 0.00 & \pm 0.01 & \pm 0.01 & \pm 0.01 & \pm 0.00 \\
\hline \multicolumn{11}{|c|}{$\Delta \log g= \pm \sigma_{\log g}$} \\
\hline Low $T_{\text {eff }}$ & \pm 0.03 & \pm 0.01 & $\mp 0.08$ & \pm 0.04 & \pm 0.00 & \pm 0.07 & \pm 0.01 & \pm 0.07 & \pm 0.04 & \pm 0.07 \\
\hline Solar & \pm 0.00 & \pm 0.00 & \pm 0.00 & \pm 0.01 & - & \pm 0.01 & \pm 0.00 & \pm 0.01 & \pm 0.01 & \pm 0.01 \\
\hline High $T_{\text {eff }}$ & \pm 0.00 & \pm 0.00 & \pm 0.00 & \pm 0.02 & - & \pm 0.02 & \pm 0.01 & \pm 0.02 & \pm 0.02 & \pm 0.02 \\
\hline \multicolumn{11}{|c|}{$\Delta \xi_{\mathrm{t}}= \pm \sigma_{\xi_{\mathrm{t}}}$} \\
\hline Low $T_{\text {eff }}$ & $\mp 0.03$ & $\mp 0.04$ & $\mp 0.08$ & $\mp 0.08$ & $\mp 0.04$ & $\mp 0.04$ & $\mp 0.07$ & $\mp 0.03$ & $\mp 0.01$ & $\mp 0.00$ \\
\hline Solar & $\mp 0.01$ & $\mp 0.01$ & $\mp 0.01$ & $\mp 0.01$ & - & $\mp 0.01$ & $\mp 0.02$ & $\mp 0.00$ & $\mp 0.00$ & $\mp 0.00$ \\
\hline High $T_{\text {eff }}$ & $\mp 0.00$ & $\mp 0.02$ & $\mp 0.00$ & $\mp 0.02$ & - & $\mp 0.01$ & $\mp 0.03$ & $\mp 0.00$ & $\mp 0.00$ & $\mp 0.00$ \\
\hline \multicolumn{11}{|c|}{ Total error } \\
\hline Low $T_{\text {eff }}$ & \pm 0.07 & \pm 0.07 & \pm 0.16 & \pm 0.12 & \pm 0.14 & \pm 0.12 & \pm 0.08 & \pm 0.11 & \pm 0.08 & \pm 0.12 \\
\hline Solar & \pm 0.05 & \pm 0.04 & \pm 0.06 & \pm 0.05 & - & \pm 0.04 & \pm 0.03 & \pm 0.09 & \pm 0.05 & \pm 0.09 \\
\hline High $T_{\text {eff }}$ & \pm 0.08 & \pm 0.04 & \pm 0.08 & \pm 0.07 & - & \pm 0.07 & \pm 0.06 & \pm 0.06 & \pm 0.07 & \pm 0.12 \\
\hline
\end{tabular}

complete sample. Therefore, we decided not to remove any of these hot stars from our sample of Ba abundances.

\subsection{Ce abundances}

Cerium abundances are based on four ionized lines. The atomic parameters for these lines were initially taken from VALD3 database; however, the $\log g f$ values provided there produced a high dispersion in abundances among the lines, thus we decided to take the calibrated $\log g f$ values from Reddy et al. (2003). The lines at $4523 \AA$ and $4773 \AA$ become very blended for stars cooler than $5300 \mathrm{~K}$; therefore, we used only these lines for hotter stars (see Fig. 7).

\subsection{Nd abundances}

Neodymium abundances are calculated by using $4 \mathrm{Nd}$ II lines. We considered HFS for all the lines and the atomic parameters were taken from the Gaia-ESO Survey linelist (Heiter et al. 2015). All the lines become very blended for $T_{\text {eff }}<5000 \mathrm{~K}$, and only in spectra with $S / N>500$ can we use the line at $4811 \AA$. The lines at $4989 \AA$ and $5130 \AA$ are only used at temperatures higher than $5500 \mathrm{~K}$ and $5600 \mathrm{~K}$, respectively since below these $T_{\text {eff }}$ values, the abundances show a strong upward trend with decreasing $T_{\text {eff }}$ (see Fig. 7). Finally, the line at $5092 \AA$ is only used for $T_{\text {eff }}>5000 \mathrm{~K}$.

\subsection{Eu abundances}

Europium abundances are based on the weak ionized line at $6645.13 \AA$. This line is blended with another line at $6645.35 \AA$, thus the automatic measurement was not possible for many of the stars. We selected only the stars with $S / N>200$, and measured by hand the lines which ARES could not deblend. We considered HFS for this line and the atomic parameters were taken from the Gaia-ESO Survey linelist (Heiter et al. 2015). The error due to continuum placement was calculated in the same way as for $\mathrm{Sr}$.

\subsection{Revised abundances for refractory elements and homr stars}

In the following sections we evaluate the behaviour of the previously described elements for the different populations in the Galaxy: thin disc, thick disc, and the homr stars. We note that stars with $[\mathrm{Fe} / \mathrm{H}]>-0.2$ dex and showing enhancement in $\alpha$-element abundances were first classified as members of a homr population in Adibekyan et al. (2011, 2013). In this work we are presenting updated values of $\log g$ and $T_{\text {eff }}$ for many stars of our sample, and so we have to re-derive the abundances of refractory elements, especially those of MgI, SiI, TiI, and TiII in order to have a coherent definition of homr stars. Moreover, we have improved our linelist using the Van der Waals damping constants, and the final abundance for each element is obtained from the WM of all the available individual abundances. In Fig. 9 we can see the new $\alpha$ abundances in our sample and which stars now have a different classification. Most of these stars (24 out of 43) have $T_{\text {eff }}<5200 \mathrm{~K}$ and their abundance change can be explained by the change of $T_{\text {eff }}$ since $\log g$ variations hardly affect neutral species. Nevertheless, the difference in alpha abundances between the old and updated values is lower than 0.05 dex. 

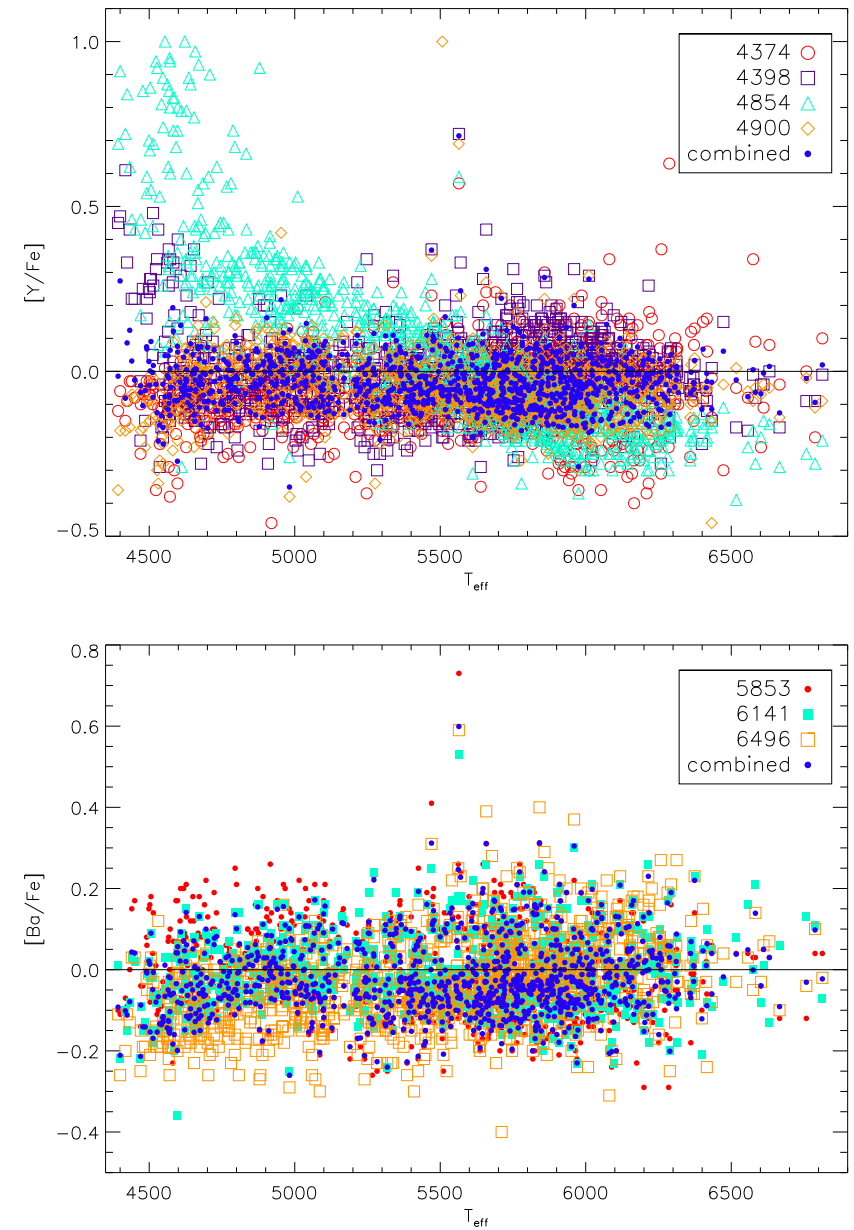

Fig. 5. Upper panel: $[\mathrm{Y} / \mathrm{Fe}]$ for three of the lines we use, together with the discarded line (blue triangles) and the final combined abundance from the six chosen lines. Lower panel: $[\mathrm{Ba} / \mathrm{Fe}]$ for each line as a function of $T_{\text {eff }}$ together with the final adopted values for each star (blue filled circles).

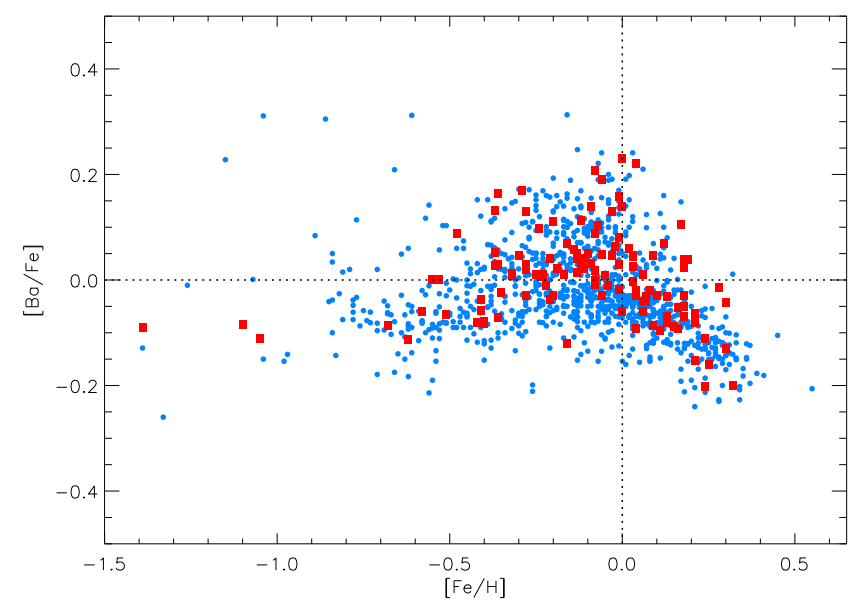

Fig. 6. Final $[\mathrm{Ba} / \mathrm{Fe}]$ as a function of $[\mathrm{Fe} / \mathrm{H}]$ for the full sample. Stars hotter than $6100 \mathrm{~K}$ are shown with red squares.

In summary, there are 10 stars that first belonged to the thin disc population and now are considered as hamr. Six of these stars have $T_{\text {eff }}<5200 \mathrm{~K}$ and the difference in $[\alpha / \mathrm{Fe}]$ is $0.004 \pm 0.005 \mathrm{dex}$ (in the sense new-old) for the 10 stars. On the other hand, 25 stars first considered as homr now belong to the thin disc. Eleven of these stars are cool and
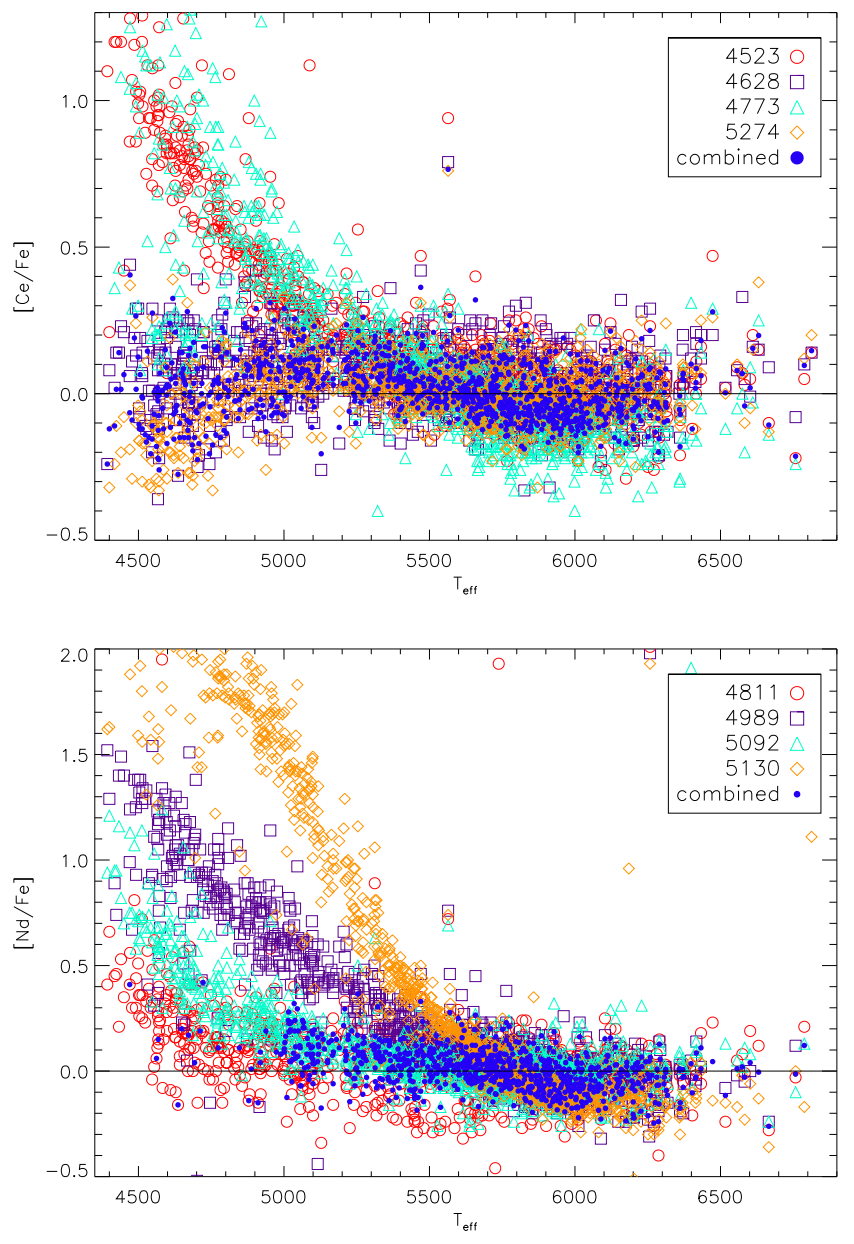

Fig. 7. $[\mathrm{Ce} / \mathrm{Fe}]$ and $[\mathrm{Nd} / \mathrm{Fe}]$ for each line as a function of $T_{\text {eff }}$ together with the final adopted values for each star (blue filled circles).

the difference in $[\alpha / \mathrm{Fe}]$ is $-0.047 \pm 0.021 \mathrm{dex}$ for the 25 stars. Finally, 50 stars remain classified as homr and in total we have 60 stars belonging to this population. For thin disc stars at $[\mathrm{Fe} / \mathrm{H}] \geq-0.2 \mathrm{dex},\langle[\alpha / \mathrm{Fe}]\rangle=0.014 \pm 0.028 \mathrm{dex}$; instead, for $h \alpha m r$ stars, $\langle[\alpha / \mathrm{Fe}]\rangle=0.095 \pm 0.029$ dex. Despite the new classification of some of our stars the separation between the two populations still exists and the difference in $[\alpha / \mathrm{Fe}]$ is above the errors (the average error of $[\alpha / \mathrm{Fe}]$ are $0.037,0.017$, and $0.031 \mathrm{dex}$ for the groups of cool, solar, and hot stars as defined in Sect. 3.1). The updated abundances for these elements are also provided in Table 3.

\section{Results and discussion}

\section{1. $[\mathrm{X} / \mathrm{Fe}]$ ratios for different stellar populations}

In Fig. 10 we show the $[\mathrm{X} / \mathrm{Fe}]$ ratios of all our elements for stars hotter than $5300 \mathrm{~K}$. We decided to study this subsample of hotter stars since at lower $T_{\text {eff }}$ the abundances present higher uncertainties. We also discarded stars with spectra of $S / N<100$ to ensure more precise measurements. For this subsample we have 539 thin disc stars, 80 thick disc stars, 28 homr stars, and 6 halo stars. We used a chemical criterion based on the $[\alpha / \mathrm{Fe}]$ ratio to separate the stellar populations, as was done in Adibekyan et al. (2011, 2012); instead, we used kinematics to select the halo stars. The corresponding $[\mathrm{X} / \mathrm{Fe}]$ ratios for the full sample are shown in Fig. A.1 together with GCE models from Romano et al. (2010) and Bisterzo et al. (2017), which are discussed in the next 

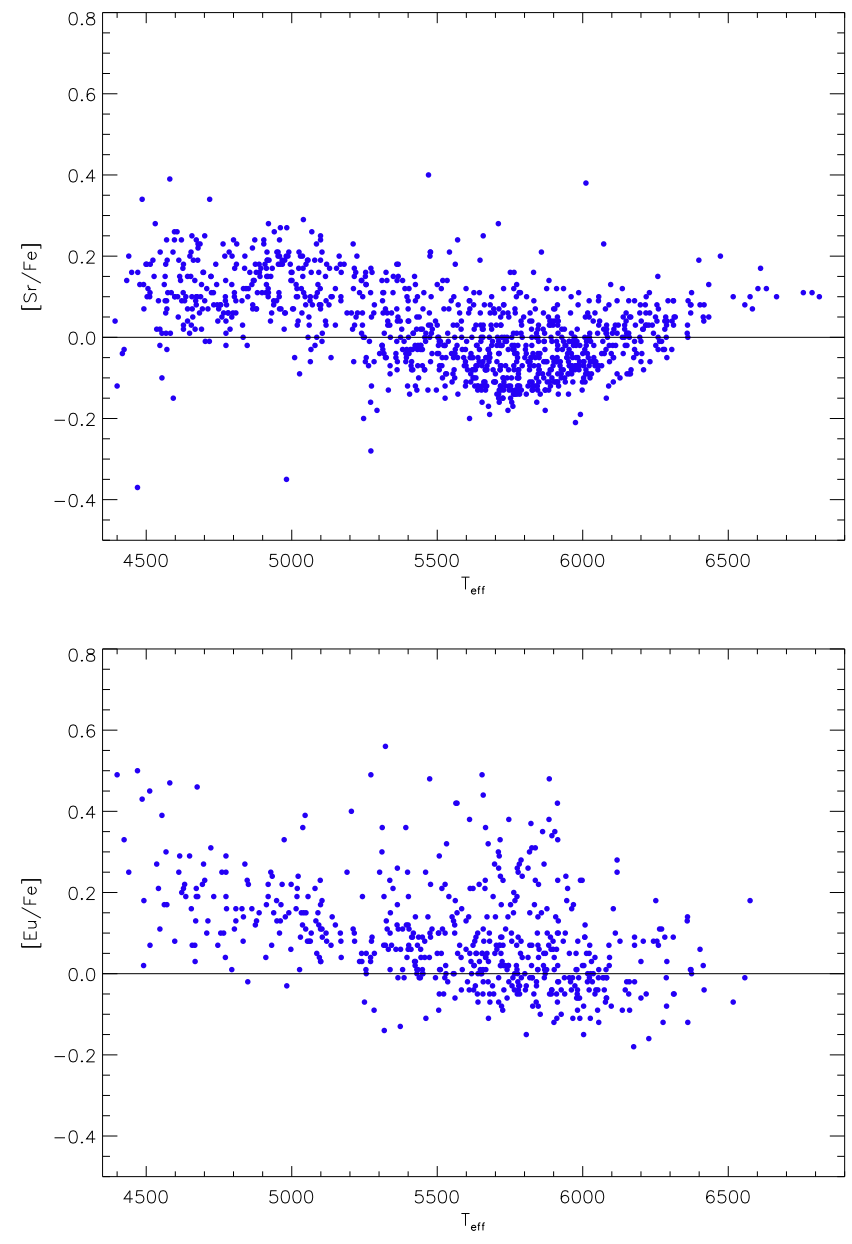

Fig. 8. $[\mathrm{Sr} / \mathrm{Fe}]$ and $[\mathrm{Eu} / \mathrm{Fe}]$ as a function of $T_{\text {eff }}$.

paragraphs. We can see in this plot that the scatter of abundances is quite high, especially among the thick disc stars. However, this scatter is, in general, higher than the $2 \sigma$ errors suggesting that the spread is real (for the coolest stars the typical $1 \sigma$ errors are around 0.10 dex, see Table 4). The abundances of Eu show very high errors, probably overestimated for most of the stars, due to the strong effect of the continuum placement uncertainty on the small EWs of the Eu line used here. We note that the star HD 11397 shows very high abundances $([\mathrm{X} / \mathrm{Fe}] \gtrsim 0.7)$ for the $s$-process elements $\mathrm{Sr}, \mathrm{Y}, \mathrm{Zr}, \mathrm{Ba}, \mathrm{Ce}$, and $\mathrm{Nd}$. However, it is not enhanced in the $r$-process element Eu. This star was already discussed in Pompéia \& Allen (2008) who reported that it has an abundance profile similar to the mild Ba stars. We also find three thick disc stars with $-0.61<[\mathrm{Fe} / \mathrm{H}]<-0.32$ (HD 126803, CD-436810 and HD 28701) that show enhanced abundances of $\mathrm{Sr}, \mathrm{Y}$, and $\mathrm{Zr}$ when compared to other thick disc stars of similar metallicity. Two of them also show enhancement of $\mathrm{Ba}, \mathrm{Ce}$, and $\mathrm{Nd}$, although the level of enhancement is not high enough for them to be considered $s$-enriched stars.

In Figs. 3-8 we can see that the most evident trends of abundances with $T_{\text {eff }}$ take place in cool stars. However, at high temperatures some elements also show slight trends with $T_{\text {eff }}$. This is the case of $[\mathrm{Cu} / \mathrm{Fe}]$, which barely presents positive values at $T_{\text {eff }} \gtrsim 6000 \mathrm{~K}$, or $[\mathrm{Zn} / \mathrm{Fe}]$, which also displays a decreasing trend for the hotter stars. On the other hand, $[\mathrm{Sr} / \mathrm{Fe}]$ tends to increase at higher $T_{\text {eff }}$. Therefore, to avoid the systematic effects associated with the errors on stellar parameters we decided to study the differences in abundances among the different

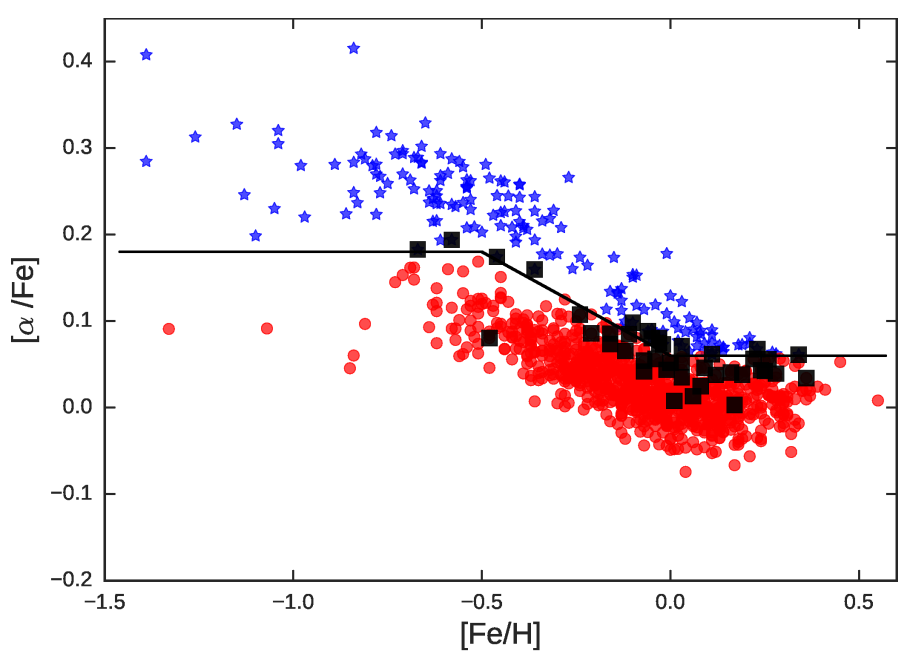

Fig. 9. Updated abundances of $\alpha$ elements (mean abundance of $\mathrm{Si}, \mathrm{Mg}$, and $\mathrm{Ti}$ ) with the new stellar parameters presented in this work. Thin disc stars (red circles) and thick disc stars (blue stars) are chemically separated by their $\alpha$ content (thick line). The hamr stars are the prolongation of thick disc stars at $[\mathrm{Fe} / \mathrm{H}]>-0.2$ dex. The stars with a different classification with respect to Adibekyan et al. (2011) are shown as black squares.

populations by using only stars with $T_{\odot} \pm 300 \mathrm{~K}$, as was done in Adibekyan et al. (2012). This subsample is composed of 328 thin disc stars, 49 thick disc stars, 16 homr stars and 4 halo stars (see Fig. 11). We also plot the average abundance in each 0.1 dex metallicity bin for the different components. The population of hamr stars (shown with red squares) is well mixed with the thin disc for most of the elements; however, it shows a clear average enhancement for $\mathrm{Cu}$ and $\mathrm{Zn}$ following the behaviour of the thick disc stars. Moreover, they have $\mathrm{Y}$ and $\mathrm{Ba}$ abundances that are lower on average than those of thin disc stars. Finally, it is not surprising that they also show higher Eu on average which is mainly produced in SNe II as alpha elements.

First elements beyond the iron-peak $(\mathrm{Cu}, \mathrm{Zn})$ : Copper and zinc are transition elements between the Fe-peak and the neutron capture elements. Copper was initially thought to be mainly produced by the weak $s$-process (Sneden et al. 1991), but this view has been debated over the years; it has been suggested that $\mathrm{SNe}$ Ia would also contribute to the production of $\mathrm{Cu}$ and Zn (e.g. Matteucci et al. 1993; Mishenina et al. 2002). However, Romano \& Matteucci (2007) ruled out the contribution from $\mathrm{SNe}$ Ia and showed that explosive nucleosynthesis in corecollapse $\mathrm{SNe}$ is only important at very low metallicities, hence $\mathrm{Cu}$ is mostly produced by the weak $s$-process. Moreover, other works claim that only $5 \%$ of $\mathrm{Cu}$ is provided by the main- $s$ component and the remaining can be explained by $\mathrm{SNe}$ II yields from different masses and metallicities, i.e. the weak- $s$ component (Travaglio et al. 2004b; Bisterzo et al. 2005). The weak $s$ process occurs in massive stars during core $\mathrm{He}$ and shell $\mathrm{C}$ burning, where neutrons are provided by the ${ }^{22} \mathrm{Ne}(\alpha, n)^{25} \mathrm{Mg}$ reaction, and partly produces neutron capture isotopes lighter than $A \sim 90$ (e.g. Pignatari et al. 2010, and references therein). The weak $s$-process is considered to be of secondary nature, since the neutron source, ${ }^{22} \mathrm{Ne}$, originates from pre-existing CNO nuclei, and thus depends on the initial metallicity of the star.

In Fig. 11 we can see that $[\mathrm{Cu} / \mathrm{Fe}]$ diminishes monotonically for lower metallicities in the thick disc population and the few halo stars of our sample (as already seen in halo stars by Sneden et al. 1991). This is in agreement with a $\mathrm{Cu}$ 


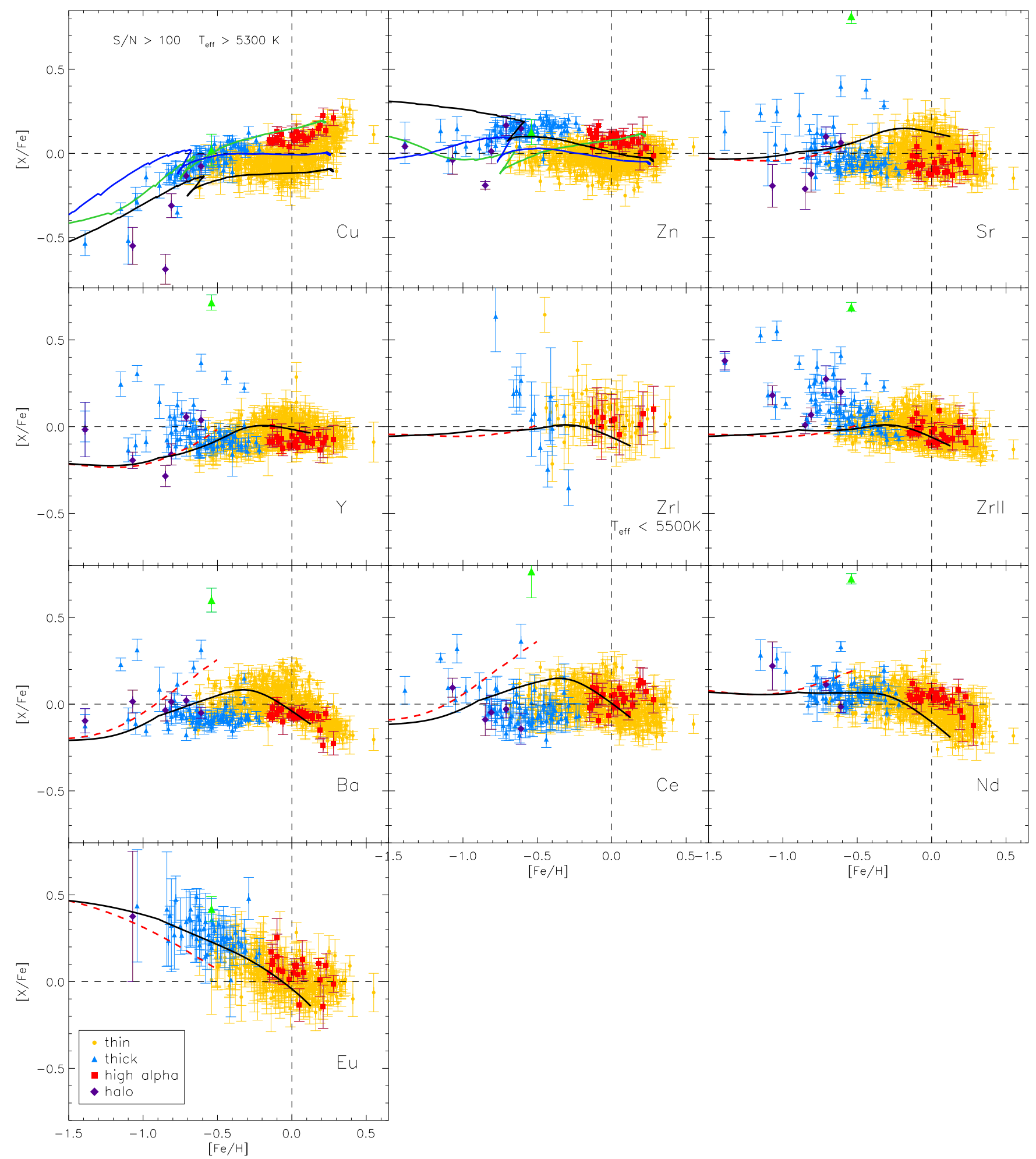

Fig. 10. Final $[\mathrm{X} / \mathrm{Fe}]$ ratios as a function of $[\mathrm{Fe} / \mathrm{H}]$ for stars with $T_{\text {eff }}>5300 \mathrm{~K}$ and $S / N>100$. The different stellar populations are depicted with different colours and symbols as explained in the legend. The green bigger triangle is the $s$-enriched star HD 11397 . For $\mathrm{Cu}$ and $\mathrm{Zn}$ we overplot the GCE models 1, 4, and 5 (green, blue, and black lines, respectively) from Romano et al. (2010). For the rest of the elements we show the GCE models from Bisterzo et al. (2017) for the thin disc (black lines) and the thick disc (red dashed lines).

production mostly by weak $s$-process and a primary contribution by explosive nucleosyntheis in SNe II at low metallicities (Romano \& Matteucci 2007). The maximum $\mathrm{Cu}$ for thick disc stars is reached at $[\mathrm{Fe} / \mathrm{H}] \sim-0.4$ dex in agreement with the compilation made by Pignatari et al. (2010) to then steadily decrease towards lower $[\mathrm{Fe} / \mathrm{H}]$ and keep more or less constant at $-0.4<[\mathrm{Fe} / \mathrm{H}] \lesssim-0.2$. Thick disc stars have, on average, higher $\mathrm{Cu}$ abundances than thin disc stars in the metallicity region $-0.5 \lesssim[\mathrm{Fe} / \mathrm{H}] \lesssim-0.2$ as suggested in Reddy et al. (2006), Israelian et al. (2014), Yan et al. (2015), Mikolaitis et al. (2017). Although thick disc stars still present higher mean abundances on average, for lower metallicities the differences are very small 


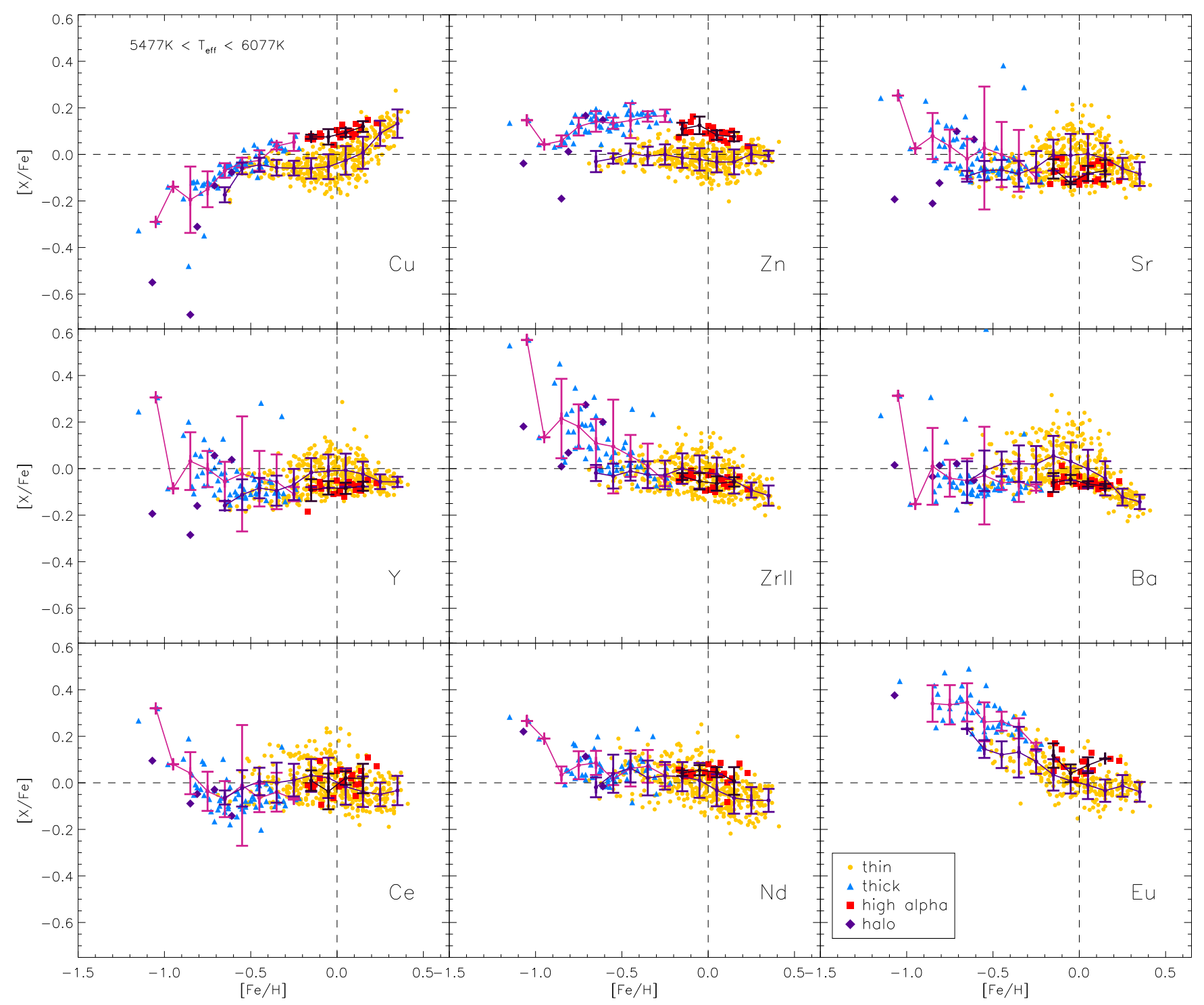

Fig. 11. Final $[\mathrm{X} / \mathrm{Fe}]$ ratios as a function of $[\mathrm{Fe} / \mathrm{H}]$ for stars with $T_{\text {eff } \odot} \pm 300 \mathrm{~K}$. The different stellar populations are depicted with different colours and symbols as explained in the legend. The mean abundances in each metallicity bin of 0.1 dex are shown together with the standard deviation.

and within the errors. On the contrary, thin disc stars present a very slight increase in abundances for $-0.8<[\mathrm{Fe} / \mathrm{H}] \lesssim 0.1$, but at super-solar metallicities there is a steep rise with respect to iron, which was first reported by Allende Prieto et al. (2004) and further confirmed here with a larger sample. Also, this increase in $\mathrm{Cu}$ abundances is found in the large sample of the AMBRE project (Mikolaitis et al. 2017). The hamr population presents the same behaviour as thin disc stars, but interestingly the abundances form an upper envelope to the thin disc, making a continuation of the thick disc, and the mean values in each metallicity bin are totally disjunct. To test whether the differences in $[\mathrm{Cu} / \mathrm{Fe}]$ ratios are significant among the different populations we have performed several Kolmogorov-Smirnov (K-S) tests whose results are shown in Table 5. In the case of $\mathrm{Cu}$, the K-S test fails to reject the hypothesis that thick disc stars and thin disc stars at $[\mathrm{Fe} / \mathrm{H}]<-0.2$ belong to the same population. On the contrary, the $\mathrm{K}-\mathrm{S}$ test rejects the hypothesis that $\mathrm{Cu}$ abundances of homr stars and thin disc stars (at $[\mathrm{Fe} / \mathrm{H}] \geq-0.2)$ are drawn from the same parent population with $p$-values lower than $10^{-10}$ both for the full sample or the subsample of stars with $T_{\odot} \pm 300 \mathrm{~K}$. In order to consider the errors of the abundance ratios, we have created 1000 samples of randomly selected abundances assuming a Gaussian distribution for each $[\mathrm{Cu} / \mathrm{Fe}]$ value, where the central value is given by the abundance ratio and sigma is equal to the error on the abundance. This test shows that for all the randomly generated samples the $p$-value of each K-S test is always lower than $2.5 \times 10^{-5}$. We note that the separation among thin disc stars and $h \alpha m r$ stars was based on $[\alpha / \mathrm{Fe}]$ ratios by Adibekyan et al. (2011), who also showed that the hamr stars are older on average than thin disk stars and have intermediate orbits between the thin and the thick disk stars. Therefore, the tests that we have performed here serve to show that the hamr stars also have different abundances of other elements apart from $\alpha$ elements, but they do not serve as a probe to distinguish them from thin disc stars.

In Fig. 10 we show the GCE models using different yields computed by Romano et al. (2010). The model that better matches our abundances is Model 1 (green line), which considers 
Table 5. Results from the K-S tests for the different $[\mathrm{X} / \mathrm{Fe}]$ abundance ratios.

\begin{tabular}{|c|c|c|c|c|c|c|c|c|}
\hline \multirow[b]{3}{*}[\mathrm{X}/\mathrm{Fe}]{} & \multicolumn{4}{|c|}{ Full sample } & \multicolumn{4}{|c|}{$T_{\odot} \pm 300 \mathrm{~K}$} \\
\hline & \multicolumn{2}{|c|}{$\begin{array}{c}\text { Thin-Thick } \\
{[\mathrm{Fe} / \mathrm{H}]<-0.2}\end{array}$} & \multicolumn{2}{|c|}{$\begin{array}{c}\text { Thin-homr } \\
{[\mathrm{Fe} / \mathrm{H}] \geq-0.2}\end{array}$} & \multicolumn{2}{|c|}{$\begin{array}{c}\text { Thin-Thick } \\
{[\mathrm{Fe} / \mathrm{H}]<-0.2}\end{array}$} & \multicolumn{2}{|c|}{$\begin{array}{c}\text { Thin-homr } \\
{[\mathrm{Fe} / \mathrm{H}] \geq-0.2}\end{array}$} \\
\hline & $p$-value & $D$ & $p$-value & $D$ & $p$-value & $D$ & $p$-value & $D$ \\
\hline $\mathrm{Cu}$ & $2.3 e-3$ & 0.21 & $1.3 e-22$ & 0.67 & $2.5 \mathrm{e}-2$ & 0.24 & $3.7 \mathrm{e}-11$ & 0.75 \\
\hline $\mathrm{Zn}$ & $3.6 e-37$ & 0.74 & $5.1 \mathrm{e}-15$ & 0.54 & $1.8 \mathrm{e}-23$ & 0.83 & $5.0 \mathrm{e}-16$ & 0.91 \\
\hline $\mathrm{Sr}$ & $4.4 \mathrm{e}-1$ & 0.10 & $1.6 e-2$ & 0.21 & $5.4 \mathrm{e}-4$ & 0.33 & $3.4 \mathrm{e}-4$ & 0.45 \\
\hline $\mathrm{Y}$ & $7.4 \mathrm{e}-2$ & 0.15 & $8.3 e-8$ & 0.39 & $3.4 \mathrm{e}-3$ & 0.29 & $5.8 \mathrm{e}-7$ & 0.58 \\
\hline ZrII & $6.8 \mathrm{e}-10$ & 0.38 & $4.8 \mathrm{e}-2$ & 0.18 & $1.8 \mathrm{e}-8$ & 0.49 & $1.5 \mathrm{e}-2$ & 0.33 \\
\hline $\mathrm{Ba}$ & $7.1 \mathrm{e}-22$ & 0.57 & $5.5 e-8$ & 0.39 & $6.4 \mathrm{e}-8$ & 0.48 & $2.1 \mathrm{e}-4$ & 0.46 \\
\hline $\mathrm{Ce}$ & $8.3 e-6$ & 0.29 & $4.8 \mathrm{e}-3$ & 0.23 & $1.2 \mathrm{e}-3$ & 0.31 & $5.5 \mathrm{e}-3$ & 0.37 \\
\hline $\mathrm{Nd}$ & $1.3 e-3$ & 0.25 & $2.2 \mathrm{e}-8$ & 0.44 & $1.5 \mathrm{e}-2$ & 0.26 & $1.0 \mathrm{e}-8$ & 0.66 \\
\hline $\mathrm{Eu}$ & $3.6 \mathrm{e}-8$ & 0.67 & $6.0 \mathrm{e}-6$ & 0.41 & $1.3 e-6$ & 0.76 & $1.2 \mathrm{e}-6$ & 0.71 \\
\hline
\end{tabular}

the case B yields for normal SNe II from Woosley \& Weaver (1995). Models 4 and 5, which consider hypernovae (HNe) yields from Kobayashi \& Nomoto (2009) with different HNe fractions, overestimate the abundances of the thick disc and underestimate the abundances on the thin disc, respectively ${ }^{7}$. Moreover, these models are not able to reproduce the increase in $\mathrm{Cu}$ at high metallicities. However, Models 4 and 5 seem to reproduce the $\mathrm{Cu}$ trends better at very low metallicities, as shown in Fig. 16 of Romano et al. (2010). Nevertheless, these authors warn about the lack of AGB yields in a full range of masses and metallicities to test their effects at low $[\mathrm{Fe} / \mathrm{H}]$.

The production of $\mathrm{Zn}$ is more complex. Half of solar $\mathrm{Zn}$ is present in the isotope ${ }^{64} \mathrm{Zn}$ which is produced by $\alpha$ freezout in neutrino winds during supernova explosions of massive stars, while all the other $\mathrm{Zn}$ isotopes are produced by the weak $s$-process (e.g. Bisterzo et al. 2005, and refererences therein). The $[\mathrm{Zn} / \mathrm{Fe}]$ ratios resemble somewhat the behaviour of alpha elements (with a flattening of abundances around solar metallicity caused by the well-known iron contribution from $\mathrm{SNe}$ Ia and clearly separated thin and thick disc); however, the growth of abundances towards lower $[\mathrm{Fe} / \mathrm{H}]$ is very slight and does not reach such high values as the alpha elements do in our $[\mathrm{Fe} / \mathrm{H}]$ range (e.g. $[\alpha / \mathrm{Fe}]>0.3 \mathrm{dex}$ at metallicity around -1 dex in Adibekyan et al. 2012, and Fig. 9). Moreover, thin disc stars present nearly flat $\mathrm{Zn}$ abundances, while alpha elements increase for lower metallicities. A maximum value of $\sim 0.2$ dex is observed for stars with $-1.0<[\mathrm{Fe} / \mathrm{H}]<-0.5$ and then slightly decrease for lower metallicities, as reported by Saito et al. (2009). The work by Mikolaitis et al. (2017) also shows this slight decrease, though it starts at lower $[\mathrm{Fe} / \mathrm{H}]$. Although thick disc stars present a plateau, the maximum $\mathrm{Zn}$ in the thick disc is observed around metallicity -0.5 dex, as reported for $\mathrm{Cu}$, but drops at higher metallicities, in contrast with $\mathrm{Cu}$. González Hernández et al. (2010), Bensby et al. (2014), Mikolaitis et al. (2017) and Duffau et al. (2017) have also found a somewhat decreasing trend with metallicity. At very low metallicities $\mathrm{Zn}$ abundances continue to increase, reaching values of $[\mathrm{Zn} / \mathrm{Fe}] \sim 0.5$ dex (e.g. Saito et al. 2009; Romano et al. 2010). This overall trend can be explained by chemical-evolution models where $\mathrm{Zn}$ is produced by $\mathrm{SNe}$ II, HNe, and $\mathrm{SNe}$ Ia with

\footnotetext{
7 The position of the models in Fig. 10 is different from the Romano et al. (2010) plots because our solar mean abundances are different from those in Grevesse \& Sauval (1998), which are the values used in the models of Romano et al. (2010).
}

various metallicities (Kobayashi \& Nomoto 2009; Saito et al. 2009), although models cannot closely match the behaviour at low metallicities.

In Fig. 11 we can also see a very well separated thick disc in all $[\mathrm{Fe} / \mathrm{H}]$ bins, as was first noted by Bensby et al. (2003) with a smaller sample and later confirmed by Mikolaitis et al. (2017). Interestingly, the homr stars also present high $\mathrm{Zn}$ abundances when compared to thin disc stars, in agreement with the $\alpha$-kind behaviour of $\mathrm{Zn}$ abundances. The K-S tests for the full sample and the solar stars reject the hypothesis that $[\mathrm{Zn} / \mathrm{Fe}]$ ratios for the thin disc and homr stars are drawn from the same population (see Table 5). A similar result is obtained for the comparison between thick disc and thin disc stars with even lower $p$-values. Also, the random generated samples always give $p$-values lower than $10^{-5}$ and $10^{-14}$ for the comparison between thin-homr and thin-thick, respectively. For a more visual comparison, we present the distribution of some abundance ratios for the stars with $T_{\odot} \pm 300 \mathrm{~K}$ in Fig. 12. Finally, in Fig. 10 we also show the same models from Romano et al. (2010) as for $\mathrm{Cu}$. Models 4 and 5 (those including $\mathrm{HNe}$ yields) reproduce better our abundances and the general lowering trend from the thick disc to the thin disc. However, in their Fig. 16, Romano et al. (2010) show that these models do not work well at low metallicities, pointing to the necessity of increasing the $\mathrm{Zn}$ yields from metal-poor core-collapse $\mathrm{SNe}$.

Light s-process elements (Sr, $Y, Z r)$ : light $s$-process elements (corresponding to the first peak) are mainly produced by AGB stars through the main $s$-process, where the required free neutrons are supplied mainly by the reaction ${ }^{13} \mathrm{C}(\alpha, n){ }^{16} \mathrm{O}$ and to a lesser extent by the reaction ${ }^{22} \mathrm{Ne}(\alpha, n)^{25} \mathrm{Mg}$. However, some studies point to a non-negligible contribution of the weak $s$-process to the production of these elements $(30 \%$ as shown in Pignatari et al. 2010). It is important to note that there is a lack of $s$-process yield calculations of AGB stars of different masses at different metallicities. Together with some uncertainties such as the convection treatment, stellar rotation, mass loss, or the size of the ${ }^{13} \mathrm{C}$ pocket; it makes the development of GCE models a difficult task (Karakas 2016). In Fig. 11 we can see that these three elements behave in a different way even though they belong to the same group. Zirconium shows a clear increasing trend for lower metallicities including halo stars, as also observed in previous works (e.g. González Hernández et al. 2010; Mishenina et al. 2013; Battistini \& Bensby 2016; Zhao et al. 2016), while Y and $\mathrm{Sr}$ present a flatter trend and most of the thick disc stars 


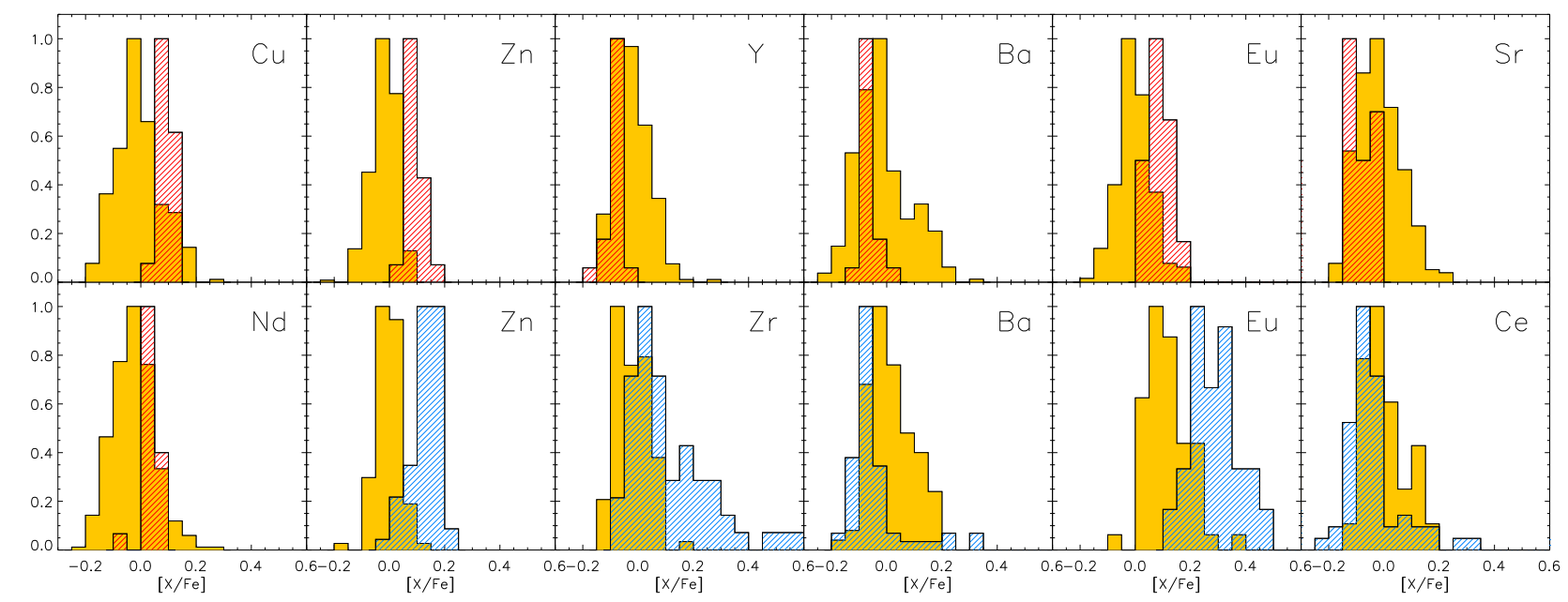

Fig. 12. Normalized distributions of several $[\mathrm{X} / \mathrm{Fe}]$ ratios for stars with $T_{\odot} \pm 300 \mathrm{~K}$. The yellow histograms represent the thin disc stars at $[\mathrm{Fe} / \mathrm{H}] \geq-0.2$ dex when compared to $h \alpha m r$ stars (red dashed histogram) or at $[\mathrm{Fe} / \mathrm{H}]<-0.2$ dex when compared to thick disc stars (blue histogram).

have subsolar abundances, similar to Bensby et al. (2014) and Zhao et al. (2016), respectively. Thick disc stars tend to increase their $\mathrm{Y}$ and $\mathrm{Sr}$ abundances from $\sim-0.1 \mathrm{dex}$ at $[\mathrm{Fe} / \mathrm{H}]=-0.2$ to $\sim 0.1 \mathrm{dex}$ at $[\mathrm{Fe} / \mathrm{H}]=-1$, but the increase is very slight when compared to $\mathrm{Zr}$. Thin disc stars present a slight rise for $\mathrm{Sr}$ and $\mathrm{Y}$ towards a maximum at $[\mathrm{Fe} / \mathrm{H}] \sim 0.0$ dex and then decrease at super-solar metallicities, while for Zr II they present a flat trend until solar metallicity and then also decrease at $[\mathrm{Fe} / \mathrm{H}] \geq 0$ dex. The group of homr stars also present subsolar abundances along $[\mathrm{Fe} / \mathrm{H}]$, as thick disc stars do, very clear for $\mathrm{Y}$ abundances. The $\mathrm{K}-\mathrm{S}$ test for $[\mathrm{Y} / \mathrm{Fe}]$ ratios provide $p$-values lower than $10^{-6}$ for the comparison of thin disc stars and homr stars (see Table 5). The K-S tests for the random generated samples considering Gaussian distributions always give $p$-values lower than 0.05 , demonstrating that $\mathrm{Y}$ abundances for both populations probably do not come from the same parent population. For the case of [ $\mathrm{Sr} / \mathrm{Fe}]$ we obtain $p$-values lower than 0.05 in $98.7 \%$ of the tests, although we note that this percentage decreases significantly when considering the full sample. In contrast, $\mathrm{Zr}$ abundances for homr stars are well mixed with those of thin disc stars, although they present a rather flat trend in contrast to the lowering trend of thin disc stars. Finally, the abundances of $\mathrm{Zr}$ for thick disc stars are clearly higher than for thin disc stars, and for all the 1000 randomly generated samples we can discard the hypothesis that both groups are drawn from the same population. The behaviour of $\mathrm{ZrI}$ abundances can be evaluated from the full sample of cool stars in Fig. A.1. Although the dispersion is higher we can still see the increasing trend of abundances as $[\mathrm{Fe} / \mathrm{H}]$ decreases, similar to the behaviour of $\mathrm{Zr}$ II. This could imply that at lower metallicities a non-negligible contribution from massive stars to $\mathrm{Zr}$ took place (see further discussion in the next section).

In Fig. 10 we also plot the GCE models for Y and Zr computed by Bisterzo et al. (2017) and for Sr (Bisterzo et al., priv. comm.). These models were built considering the contributions of $r$-process, $s$-process, and Lighter Element Primary Process (LEPP; see Sect. 4.3) ${ }^{8}$. The $r$-process contribution is only important for stars with lower metallicities as shown here. For the $s$-process contribution these authors considered AGB yields with a standard ${ }^{13} \mathrm{C}$ pocket choice. In general, these models reproduce

8 The position of the models in Fig. 10 is different from those in the Bisterzo et al. (2017) plots because our solar mean abundances are different from those in Lodders et al. (2009), which are the values used in the models of Bisterzo et al. (2017). well our observations for thin disc stars although our maximum abundances (around solar metallicity) are delayed with respect to the models (observed at $[\mathrm{Fe} / \mathrm{H}] \sim-0.25 \mathrm{dex}$ ). This delay is probably caused by the uncertainties on SNe Ia yields (which affect Fe abundances) and by different assumptions on the SFR of the models (which determine the delay of AGB stars contribution to the elements discussed here) as pointed by Bisterzo et al. (2017). On the other hand, thick disc models do not match our observations, especially for $\mathrm{Zr}$ where the model is basically flat, but our $[\mathrm{Zr} / \mathrm{Fe}]$ ratios clearly increase towards lower metallicities. Bisterzo et al. (2017) also explore the effect of different ${ }^{13} \mathrm{C}$ pocket choices for $\mathrm{Y}$ abundances, but the differences are not very great with respect to the standard case in our metallicity range.

Heavy s-process elements $(\mathrm{Ba}, \mathrm{Ce}, \mathrm{Nd})$ : these elements correspond to the second peak of $s$-process production, also known as heavy $s$-process elements. The main component of the $s$-process, namely the production by AGB stars, dominates the synthesis of these elements. In Fig. 11 we can see a clear fall of $\mathrm{Ba}$ abundances for thin disc stars from a maximum $[\mathrm{Ba} / \mathrm{Fe}] \sim 0.25$ dex at solar metallicity to $[\mathrm{Ba} / \mathrm{Fe}]<-0.2$ at super-solar metallicities, similar to the values reported by Israelian et al. (2014), Bensby et al. (2014). This decrease in abundances at high metallicities is also observed for $\mathrm{Nd}$ but not for $\mathrm{Ce}$. The decrease in $\mathrm{Ba}$ abundances at super-solar metallicities seems to be at odds with the overabundances found in young metal-rich clusters by D'Orazi et al. (2009) (see also Fig. 4 in Maiorca et al. 2012), although not all our metal-rich stars are young ${ }^{9}$. The elements $\mathrm{Ba}$ and $\mathrm{Ce}$ are mainly $s$-process in solar material $(>80 \%)$ and they show a very slight reduction in abundances from the maximum towards lower metallicities for the thin disc population. The work by Mishenina et al. (2013) reports a similar behaviour for Ce abundances, while other authors show a more flattened trend (e.g. Reddy et al. 2006; Battistini \& Bensby 2016) and Allende Prieto et al. (2004) present a strong increasing trend as $[\mathrm{Fe} / \mathrm{H}]$ decreases. On the other hand, $\mathrm{Nd}$, which is produced in a similar proportion by $r$ - and $s$-processes, presents a slight increasing trend as $[\mathrm{Fe} / \mathrm{H}]$ drops for thin disc stars, also reported by Allende Prieto et al. (2004), González Hernández et al. (2013), Mishenina et al. (2013), Battistini \& Bensby (2016), which reflects the important contribution of massive stars to this element. The thick disc stars present mostly subsolar abundances of $\mathrm{Ba}$

9 In a forthcoming work we will discuss the abundance trends with age. 
and $\mathrm{Ce}$, whereas $\mathrm{Nd}$ is super-solar and also increases towards lower metallicities. Interestingly, the homr stars show clear subsolar abundances for $\mathrm{Ba}$ as a continuation of the thick disc, resembling the behaviour of $\mathrm{Y}$. However, for $\mathrm{Nd}$, they present higher abundances on average when compared to the thin disc. We have also performed K-S tests which show that the abundances of thick and thin disc stars are probably drawn from different parent populations (see Table 12) for the case of $\mathrm{Ba}$ (all K-S tests for the random samples give $p$-values lower than $0.01)$ and for Ce $(99.8 \%$ of the tests provide $p$-values lower than 0.05). When comparing the hamr stars with the thin disc stars at $[\mathrm{Fe} / \mathrm{H}]>-0.2$ we found that $99.8 \%$ of the K-S tests applied to the random generated samples have a $p$-value lower than 0.05 .

In Fig. 10 we overplot GCE models of Ba by Bisterzo et al. (2017) and Ce and Nd (Bisterzo et al., priv. comm.). These models have the same ingredients as for light- $s$ elements. Models for the $\mathrm{Ba}$ and $\mathrm{Ce}$ abundances match the observations of the thin disc quite well, but here we observe the same delay in the maximum abundances as happens for $\mathrm{Sr}, \mathrm{Y}$, and $\mathrm{Zr}$. Our maximum abundances occur at solar metallicity, but in the models Ba and Ce peak at $[\mathrm{Fe} / \mathrm{H}] \sim-0.35 \mathrm{dex}$. On the other hand, $\mathrm{Nd}$ is better reproduced although the models keep flat at $[\mathrm{Fe} / \mathrm{H}]<-0.3 \mathrm{dex}$ even though the abundances slightly increase towards lower metallicities ${ }^{10}$. As happens for light- $s$ elements, any of the models for the thick disc seem to reproduce our observations. However, we have to consider the lack of stars at $[\mathrm{Fe} / \mathrm{H}]<-0.8 \mathrm{dex}$ in our sample. By filling these regions with more stars we might have different trends for the thick disc than are observed here.

The r-process element Eu: Europium is the only element in our work with a dominant contribution by the $r$-process. Its steep increase in abundance towards low metallicities resembles the behaviour of $\alpha$-elements. Therefore, due to the unknown mechanisms of Eu production, GCE models have considered that it is synthesized by a primary process in massive stars exploding as SNe II (e.g. Travaglio et al. 1999; Bisterzo et al. 2017). This trend has been extensively studied in the literature with qualitative good agreement among different authors. We find a maximum $[\mathrm{Eu} / \mathrm{Fe}]$ value of $\sim 0.5 \mathrm{dex}$ at $[\mathrm{Fe} / \mathrm{H}] \sim-0.8 \mathrm{dex}$, which then monotonically decreases towards higher metallicities and becomes flat at $[\mathrm{Fe} / \mathrm{H}]>0$ dex. All the K-S tests reject the hypothesis that Eu abundances in thick disc stars and thin disc stars are drawn from the same population ( $p$-values always lower than 0.02). In Fig. 12 we can appreciate how well separated the two populations are. Also, $97.2 \%$ of the tests provide $p$-values lower than 0.05 when comparing the populations of thin disc stars and homr stars since this last group presents higher Eu abundances, as can be expected due to the $\alpha$-like behaviour of $\mathrm{Eu}$. The GCE models by Bisterzo et al. (2017) shown in Fig. 10 match our observations very well, although they seem to underestimate the abundances of the thick disc stars ${ }^{11}$.

\subsection{Relative contribution of $r$ - and s-process elements along the chemical history of the Galaxy}

Since the $s$ - and $r$-processes are associated with stars of different masses and metallicities that eject their material to the ISM at

${ }^{10}$ We have shifted the models of Nd by +0.15 dex in Fig. 10 since our solar abundance is much higher than the solar reference of Bisterzo et al. (2017) and the models would be too low when compared with our data.

11 We have shifted the models of Eu by +0.1 dex in Fig. 10 since our solar abundance is much higher than the solar reference of Bisterzo et al. (2017) and the models would be too low when compared with our data.

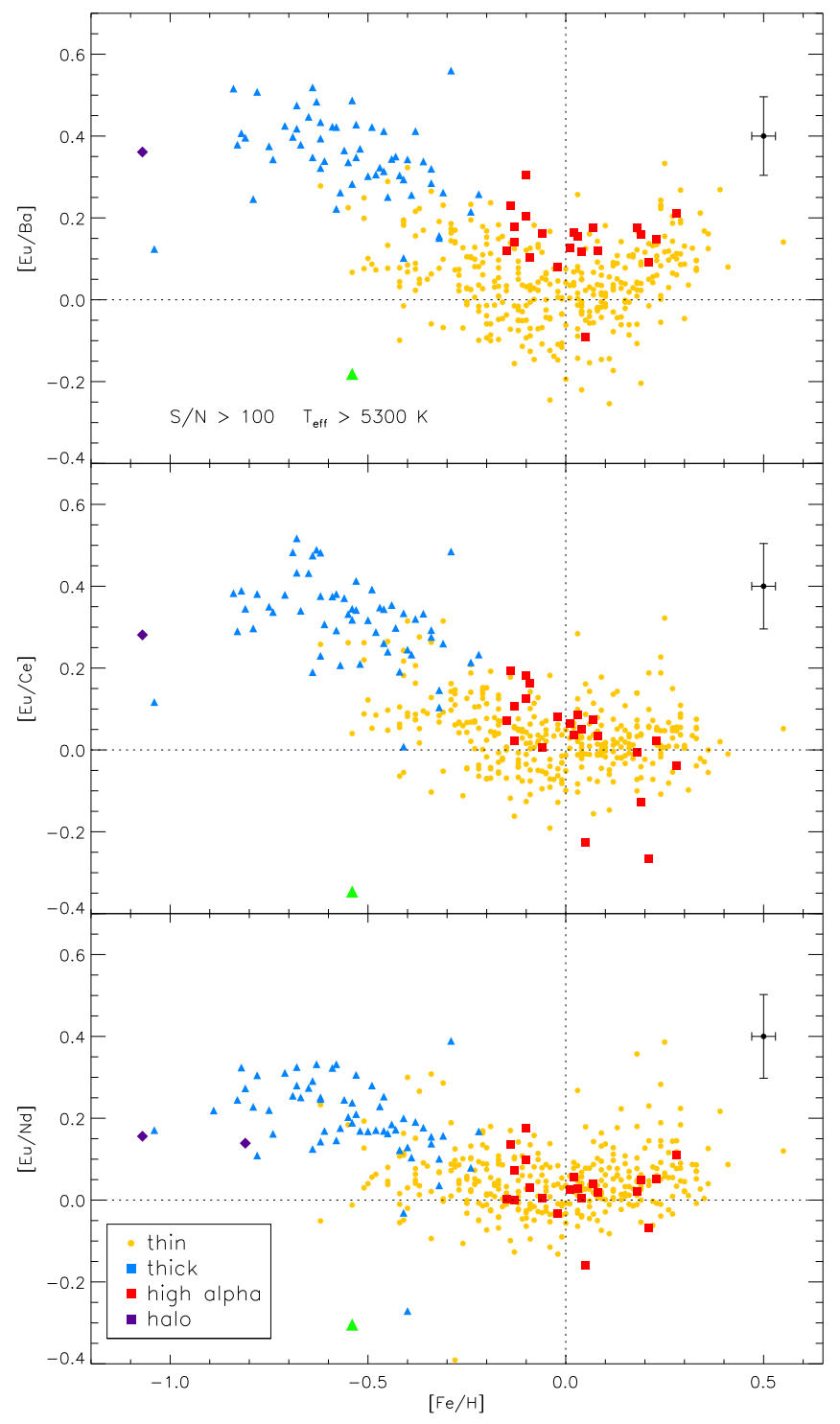

Fig. 13. Abundance ratios between the $r$-process element $\mathrm{Eu}$ and the heavy- $s$ elements as a function of $[\mathrm{Fe} / \mathrm{H}]$ for stars with $T_{\text {eff }}>5300 \mathrm{~K}$ and $S / N>100$. Symbols as in Fig. 10.

different moments of the evolution of the Galaxy, their contribution to heavy element production varies with time. Therefore, one way to disentangle the contribution of each process is to check the behaviour of abundance ratios of elements of different types. For example, the $[\mathrm{Ba} / \mathrm{Eu}]$ ratio has been extensively used to determine whether the $s$ - or $r$-process dominated the nucleosynthesis at a given moment of the evolution of the Galaxy. Early studies on very metal-poor stars reported the enrichment of Eu when compared to other $n$-capture elements (Spite \& Spite 1978 ) pointing to the importance of the $r$-process for very old stars. At very low metallicities the $r$-process is expected to dominate the production of heavy elements since massive stars were the first to explode as core-collapse SNe and enrich the ISM with their material before the AGB stars contributed to the main $s$ process. In Fig. 13 we can see how the ratios of Eu versus other heavy- $s$ elements increase towards lower metallicities. Since the contribution from $r$-process to $\mathrm{Ba}$ and $\mathrm{Ce}$ is lower $(<20 \%)$ than to $\mathrm{Nd}(\sim 45 \%)$ the ratios of $[\mathrm{Eu} / \mathrm{Ba}]$ and $[\mathrm{Eu} / \mathrm{Ce}]$ reach values up to $\sim 0.5 \mathrm{dex}$, while the maximum of $[\mathrm{Eu} / \mathrm{Nd}]$ is $\sim 0.35 \mathrm{dex}$. The 


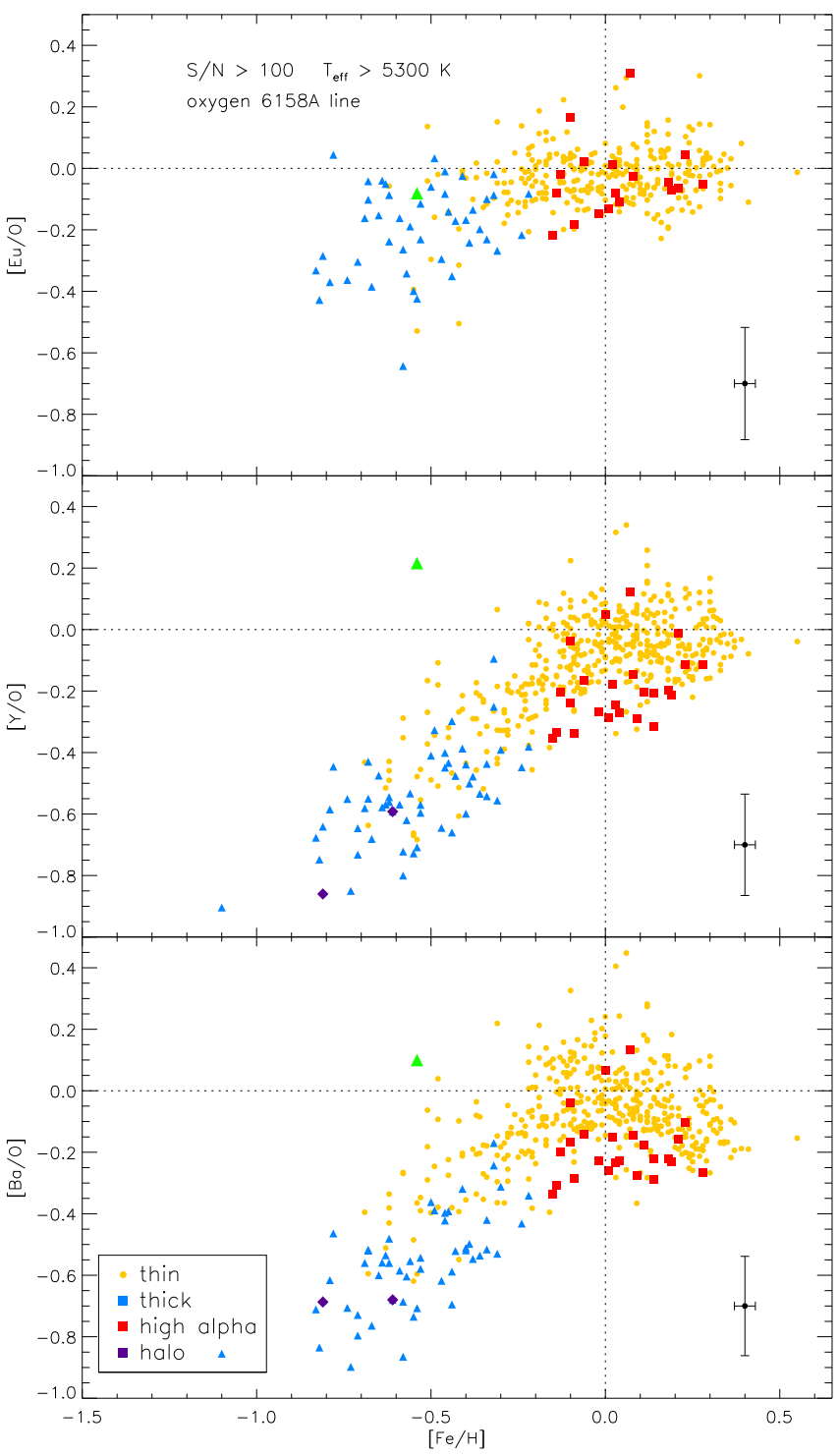

Fig. 14. Abundance ratios between the $\alpha$ element $\mathrm{O}$ and the $n$-capture elements $\mathrm{Eu}, \mathrm{Ba}$, and $\mathrm{Y}$ as a function of $[\mathrm{Fe} / \mathrm{H}]$ for stars with $T_{\text {eff }}>5300 \mathrm{~K}$ and $S / N>100$. Symbols as in Fig. 10.

minimum of these ratios is observed around solar metallicity to then grow again towards super-solar metallicities for $\mathrm{Ba}$ and $\mathrm{Nd}$, due to the continuous decrease of these heavy-s elements as the metallicity increases.

Also, by studying different ratios we can get information about the masses of the progenitors enriching the ISM at the formation time of our stars. The work by Travaglio et al. (1999) showed that the best progenitors for reproducing the $r$-process contribution to the enrichment of the Galaxy are SNe II from stars with masses $8-10 M_{\odot}$. On the other hand, more massive SNe II of $M>15 M_{\odot}$ enriched the ISM with oxygen at earlier times since those massive stars evolve faster. As a consequence we can observe that the ratios of $r$-process elements with respect to oxygen are negative for low metallicities. In Fig. 14 we show the ratios between Eu-Y-Ba and $\mathrm{O}$ using the abundances of the oxygen line at $6158 \AA$ derived for the same sample by Bertran de Lis et al. (2015). Since we do not have very metal-poor stars we cannot observe the behaviour of $Y$ and $\mathrm{Ba}$ at very low metallicities, where they are thought to be mainly produced by the $r$ - and not the $s$-process. We can see

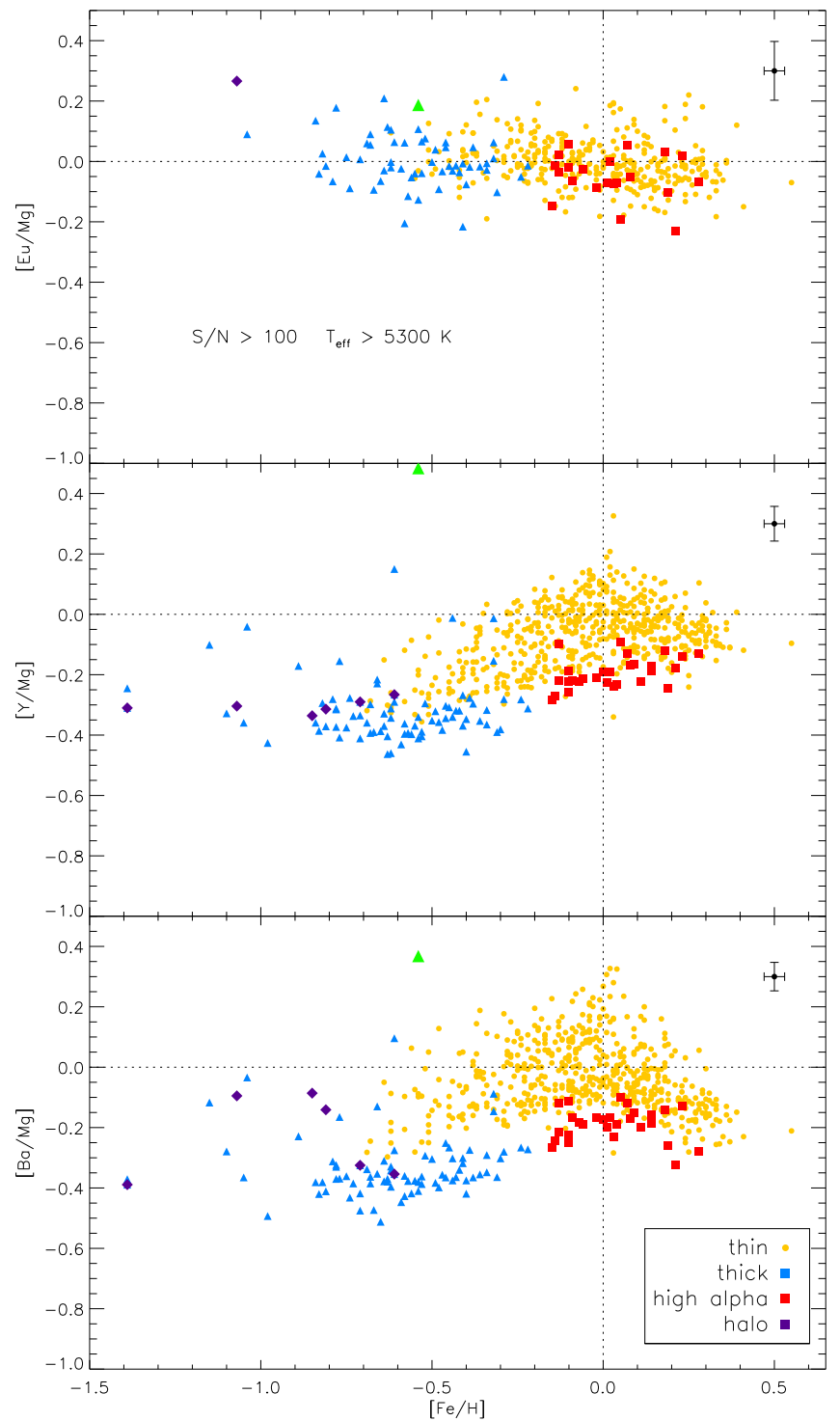

Fig. 15. Abundance ratios between the $\alpha$ element $\mathrm{Mg}$ and the $n$ capture elements $\mathrm{Eu}, \mathrm{Ba}$, and $\mathrm{Y}$ as a function of $[\mathrm{Fe} / \mathrm{H}]$ for stars with $T_{\text {eff }}>5300 \mathrm{~K}$ and $S / N>100$. Symbols as in Fig. 10.

how $[\mathrm{Eu} / \mathrm{O}]$ has a less steep decline towards lower metallicities when compared to $\mathrm{Ba}$ and $\mathrm{Y}$. This occurs because $\mathrm{Eu}$ is a pure $r$-process, whereas $\mathrm{Y}$ and $\mathrm{Ba}$ at $[\mathrm{Fe} / \mathrm{H}] \sim-1$ dex are mainly produced by AGB stars, which evolve more slowly than the progenitors of Eu and present an even longer delay with respect to the more massive progenitors of oxygen. At this point it is also interesting to compare our heavy elements with $\mathrm{Mg}$, another $\alpha$ element, using the rederived abundances in this work. In Fig. 15 we can see the same decreasing trends towards lower metallicities for $\mathrm{Y}$ and $\mathrm{Ba}$ but less steep than when comparing oxygen, while $[\mathrm{Eu} / \mathrm{Mg}]$ is mostly flat. This might be explained by increasing $\mathrm{O} / \mathrm{Mg}$ yields for higher mass $\mathrm{SNe}$ progenitors (e.g. Woosley \& Weaver 1995; McWilliam et al. 2008, and references therein). Thus, the production of oxygen would start earlier in the Galaxy producing higher $[\mathrm{O} / \mathrm{Mg}]$ at lower $[\mathrm{Fe} / \mathrm{H}]$. Moreover, the $[\mathrm{Eu} / \mathrm{Mg}]$ is mostly flat suggesting that these two elements receive an important contribution from $\mathrm{SNe}$ progenitors of similar masses but less massive than oxygen progenitors, as explained above. However, the study of McWilliam et al. (2008) discarded the possibility of increasing $\mathrm{O} / \mathrm{Mg}$ yields for higher mass $\mathrm{SNe}$ 


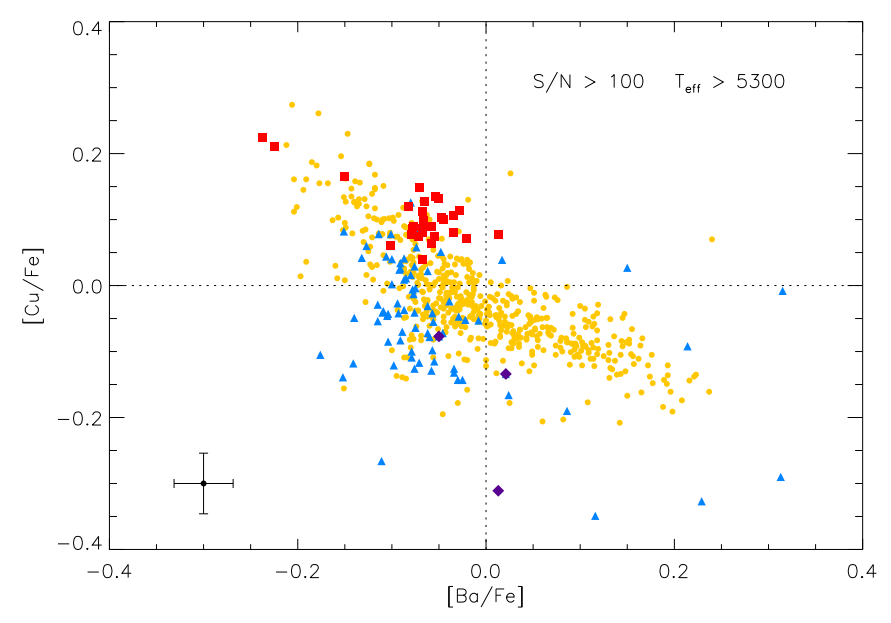

Fig. 16. $[\mathrm{Cu} / \mathrm{Fe}]$ as a function of $[\mathrm{Ba} / \mathrm{Fe}]$ for stars with $T_{\text {eff }}>5300 \mathrm{~K}$ and $S / N>100$. Symbols as in Fig. 10.

progenitors since it would imply a metallicity-dependent initial mass function (IMF), that is, an increase in the fraction of low-mass $\mathrm{SNe}$ at higher $[\mathrm{Fe} / \mathrm{H}]$. Instead, they proposed that a metallicity-dependent modulation of the $\mathrm{SNe} \mathrm{O} / \mathrm{Mg}$ ratio can perfectly explain the behaviour of this ratio in the disc and in the bulge. In Fig. 15 it is also interesting to see the well-defined separation of thick disc and homr stars with respect to the thin disc group for $[\mathrm{Y} / \mathrm{Mg}]$ and $[\mathrm{Ba} / \mathrm{Mg}]$.

In Fig. 16 we show the correlation of $[\mathrm{Cu} / \mathrm{Fe}]$ with $[\mathrm{Ba} / \mathrm{Fe}]$ abundances, which is quite obvious for thin disc stars but does not seem to hold for the other populations. This correlation was first found by Castro et al. (1999) using a small sample of metal-rich stars in the Ursa Major moving group. They pointed to a possible anticorrelation between $\mathrm{Cu}$ and $s$-process elements perhaps caused by $\mathrm{Cu}$ acting as seed for $n$-capture elements. However, we do not find such a tight correlation with any of the other heavy-s or light- $s$ elements. Later, the work by Allen \& Porto de Mello (2011) did not find a $\mathrm{Cu}-\mathrm{Ba}$ correlation in a sample of barium and normal stars, while $\mathrm{Zn}$ showed increasing trends with $\mathrm{Ba}, \mathrm{Sr}, \mathrm{Y}, \mathrm{Nd}$, and tighter increasing trends with $\mathrm{Eu}$, and other $r$-elements (Gd and Dy); the authors conclude that the $r$-process is contributing to $\mathrm{Zn}$ production with a higher proportion than to $\mathrm{Cu}$ for those stars. In contrast to those trends with $s$-elements in Allen \& Porto de Mello (2011), our thin disc stars present lower $\mathrm{Zn}$ abundances as $\mathrm{Ba}$ and especially $\mathrm{Sr}$ increase, but in a less tight way than $\mathrm{Cu}$. On the other hand, we also find slightly higher abundances of $\mathrm{Zn}$ as Eu increases, while $\mathrm{Cu}$ presents a flattened trend suggesting that $\mathrm{Zn}$ receives a major contribution from SNe II. This is in agreement with current nucelosynthesis models where $\mathrm{Zn}$ receives an important contribution of neutrino winds during $\mathrm{SNe}$ explosions of massive stars. Nevertheless, the dispersion in these correlations is too high to draw any firm conclusion.

\subsection{Ratio of heavy-s to light-s elements}

The main component of the $s$-process produced in low-mass AGB stars $\left(1.3 M_{\odot} \lesssim M \lesssim 3.5 M_{\odot}\right)$ reproduces most of the $s$-only isotopes in the solar system. The $n$-capture takes place in the He intershell where ${ }^{56} \mathrm{Fe}$ nuclei are fed by neutrons produced in the reaction ${ }^{13} \mathrm{C}(\alpha, n){ }^{16} \mathrm{O}$ during the interpulse phase of thermally pulsing AGB stars (TP-AGB; e.g. Travaglio et al. 2004a). This reaction releases a low neutron density $\left(<10^{8} \mathrm{n} / \mathrm{cm}^{3}\right)$ and

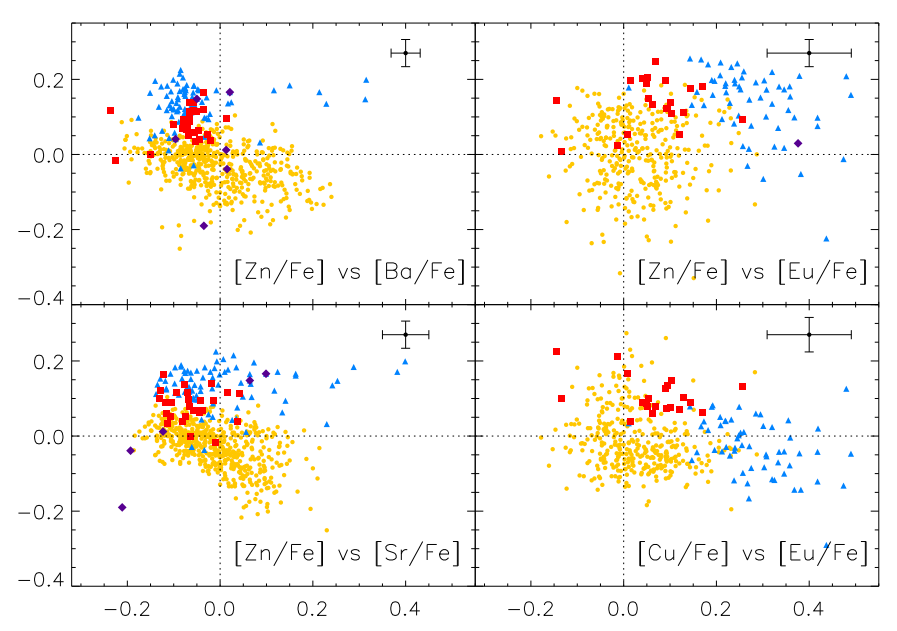

Fig. 17. Several abundance ratios for stars with $T_{\text {eff }}>5300 \mathrm{~K}$ and $S / N>100$. Symbols as in Fig. 10.

needs a lower temperature than the ${ }^{22} \mathrm{Ne}(\alpha, n)^{25} \mathrm{Mg}$ reaction (e.g. Travaglio et al. 2004a; Fishlock et al. 2014; Bisterzo et al. 2016), which is only partially activated during the convective thermal pulses in this kind of stars. Also, the elements produced in this way may depend on the size of the ${ }^{13} \mathrm{C}$ pocket (e.g. Bisterzo et al. 2014), a ${ }^{13} \mathrm{C}$ rich region created by proton capture of ${ }^{12} \mathrm{C}$ during the different third dredge-up mixing episodes. Light- $s$ elements are created first and as the neutron exposure increases, the second-peak group is created. For more massive AGB stars, $M \gtrsim 4-8 M_{\odot}$, (and at halo metallicities) there is a higher amount of primary ${ }^{22} \mathrm{Ne}$ which sets off the second reaction, while the neutron source by the ${ }^{13} \mathrm{C}$ reaction is lower, due to the smaller ${ }^{13} \mathrm{C}$ pocket size of these stars. In the ${ }^{22} \mathrm{Ne}$ reaction the density of neutrons is much higher $\left(>10^{11} \mathrm{n} / \mathrm{cm}^{3}\right)$, triggering an overproduction of neutron-rich isotopes such as ${ }^{86} \mathrm{Kr}$, ${ }^{87} \mathrm{Rb}$, and ${ }^{96} \mathrm{Zr}$. However, the contribution of intermediate-mass AGB stars to heavy-s elements is not significant because their He-intershell is one order of magnitude smaller than in lowmass AGB stars with an uncertain formation of the ${ }^{13} \mathrm{C}$ pocket and a less efficient third dredge-up (e.g. Bisterzo et al. 2016). Moreover, the neutron exposure of the ${ }^{22} \mathrm{Ne}$ reaction is lower, and thus a smaller quantity of $s$-elements is expected, especially the heavier ones (e.g. Cristallo et al. 2015b). Nevertheless, intermediate-mass AGB stars can supply up to $10 \%$ to light- $s$ elements (Travaglio et al. 2004a).

Low-mass AGB stars produce a higher proportion of heavy- $s$ with respect to light- $s$ elements (evaluated with the ratio $[h s / l s]$ ) at solar metallicities and below (e.g. Karakas \& Lugaro 2016). This can be seen in Fig. 18 where we show the ratios of the three light- $s$ elements with respect to Ba, a heavy-s element. At metallicities lower than solar, thin disc stars show negative ratios, that is, they have higher abundances of the heavy-s element $\mathrm{Ba}$. However, for a given $[\mathrm{Fe} / \mathrm{H}]$, thick disc stars have less $\mathrm{Ba}$ with respect to light-s (lower $[h s / l s]$ ) than thin disc stars. This trend is probably produced because thick disc stars are older and at their time of formation less low-mass AGB stars (which evolve more slowly than intermediate-mass AGBs) have contributed to the ISM enrichment where they were formed. On the other hand, at super-solar metallicities light- $s$ elements with respect to $\mathrm{Ba}$ show an increasing trend with metallicity, very clear for [Y/Ba]. This might be caused by the contribution of metal-rich AGB stars which provide higher abundances of light-s elements such 


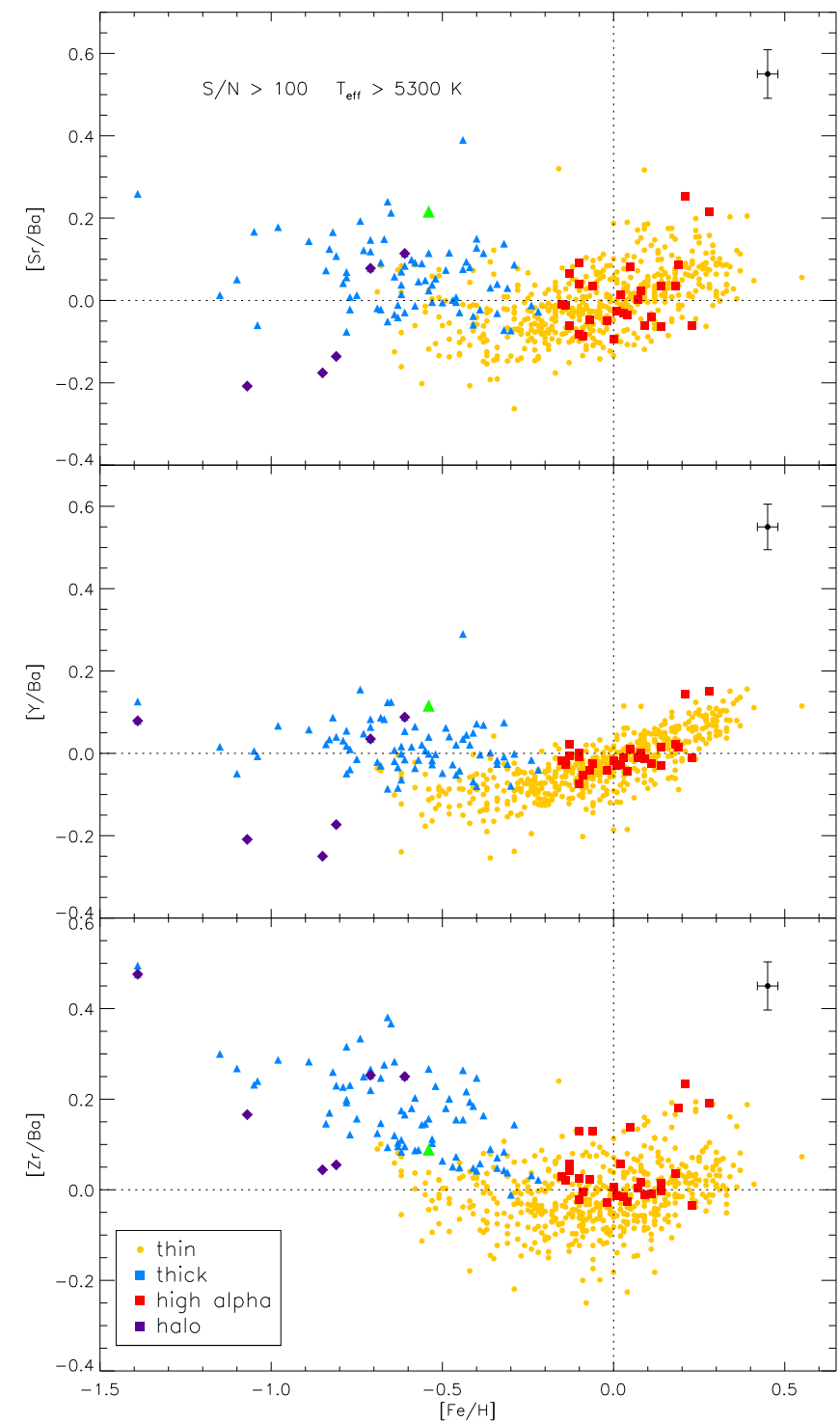

Fig. 18. Abundance ratios of light- $s$ elements with respect to the heavy$s$ element $\mathrm{Ba}$ as a function of $[\mathrm{Fe} / \mathrm{H}]$ for stars with $T_{\text {eff }}>5300 \mathrm{~K}$ and $S / N>100$. Symbols as in Fig. 10.

as $\mathrm{Y}$ and $\mathrm{Sr}$ than heavy-s elements like Ba or Ce (see Fig. 10 in Karakas \& Lugaro 2016).

This behaviour can also be observed by evaluating the ratio of heavy-s to light- $s$ in Fig. 19. In this plot $h s$ is the average abundance of $\mathrm{Ba}, \mathrm{Ce}$, and $\mathrm{Nd},{ }^{12}$ while $l s$ is the average abundance of $\mathrm{Sr}, \mathrm{Y}$, and $\mathrm{Zr}$. This ratio is rather flat for thin disc stars at low $[\mathrm{Fe} / \mathrm{H}]$, but has a maximum at $[\mathrm{Fe} / \mathrm{H}] \sim-0.4 \mathrm{dex}$ where it starts to decline towards higher metallicities. It is also at $[\mathrm{Fe} / \mathrm{H}] \sim-0.5$ dex where the production of light- $s$ elements such as $\mathrm{Y}$ in low-mass AGB stars begins to be higher than $\mathrm{Ba}$ as the metallicity increases (see Fig. 1 in Travaglio et al. 2004a). As the metallicity decreases there is a higher number of neutrons available per Fe seed; the higher neutron density allows for the build-up of heavier elements and the $[h s / l s]$ ratio is expected to increase as the metallicity diminishes (e.g. Busso et al. 1999). As

\footnotetext{
12 A similar plot can be obtained if only Ba and Ce are considered as heavy-s, with $[h s / l s]$ ratios slightly shifted towards lower values for thick disc stars. In this case, only a very small fraction of thick disc stars would show positive $[h s / l s]$ values.
}

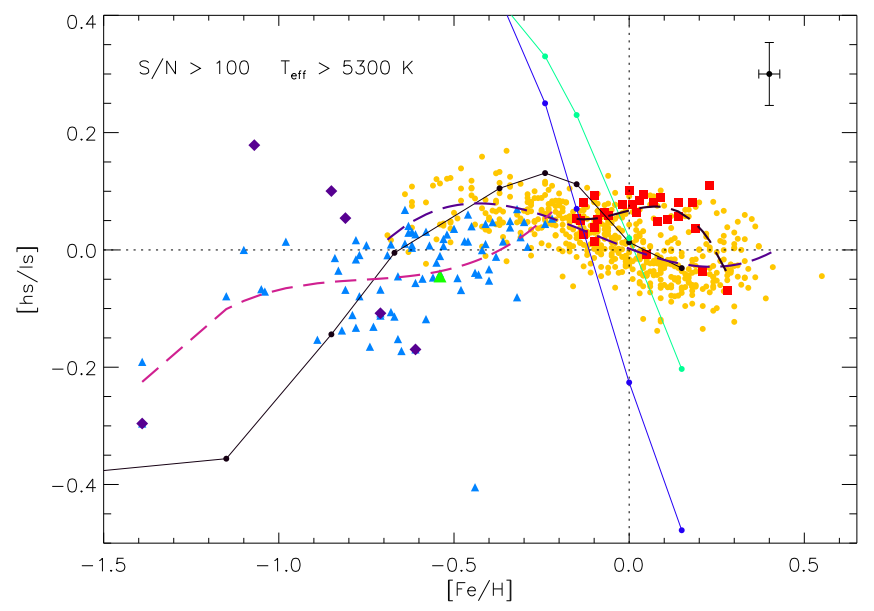

Fig. 19. Ratios of heavy-s to light- $s$ as a function of $[\mathrm{Fe} / \mathrm{H}]$ for stars with $T_{\text {eff }}>5300 \mathrm{~K}$ and $\mathrm{S} / \mathrm{N}>100$. Symbols as in Fig. 10. The blue, green, and black lines are AGB models of $2 M_{\odot}, 3 M_{\odot}$, and $6 M_{\odot}$, respectively, from Cristallo et al. (2015b). The long-dashed lines are polynomial fits to the different populations.

the metallicity decreases further, the $[h s / l s]$ ratio reaches a maximum around $[\mathrm{Fe} / \mathrm{H}]=-1$ dex (only one of our halo stars shows a high value at this metallicity), due to the progressive build-up of the third s-peak at $\mathrm{Pb}$, which becomes dominant over the $l s$ and $h s$ production (e.g. Travaglio et al. 2004a).

The rise of $[h s / l s]$ towards lower metallicities is very clear for thin disc stars; however, thick disc stars present the opposite trend: decreasing $[h s / l s]$ with decreasing $[\mathrm{Fe} / \mathrm{H}]$. This suggests that low-mass AGB stars are not the only important contribution to $s$-elements of thick disc stars. Interestingly, most of the homr stars seem to follow the trend of thick disc stars (which starts from subsolar ratios up to $[\mathrm{Fe} / \mathrm{H}] \sim-0.4 \mathrm{dex}$, and continues with super-solar ratios as the metallicity increases) and they present higher $[h s / l s]$ on average than thin disc stars at super-solar metallicities. The overabundance of light- $s$ elements with respect to heavy-s elements was noted by Travaglio et al. (2004a), but at lower metallicities than the values found here for our thick disc stars. Battistini \& Bensby (2016) also find high abundances of $\mathrm{Sr}$ and $\mathrm{Zr}$ at $[\mathrm{Fe} / \mathrm{H}] \sim-0.5$ dex. This overabundance of light- $s$ elements could not be atributed to the $r$-process or the standard weak $s$-process, so Travaglio et al. (2004a) proposed that an extra source of primary $s$-process production was contributing to light- $s$ elements at low metallicities and they called it the Lighter Element Primary Process (LEPP). In recent works, based on the analysis of $s$-only isotopes, Bisterzo et al. $(2014,2016)$ also claimed the existence of an unknown $s$-process contribution to explain the solar abundances of light- $s$ elements. In addition, Pignatari et al. (2013) proposed that a cs-component ("cold" C-burning component) could produce the missing light- $s$ elements through the ${ }^{13} \mathrm{C}(\alpha, n){ }^{16} \mathrm{O}$ reaction in massive stars (in contrast to the expected ${ }^{22} \mathrm{Ne}$ source), explaining (at least partially) the LEPP signature proposed by Travaglio et al. (2004a). On the other hand, by increasing the size of the ${ }^{13} \mathrm{C}$ pocket the models of Trippella et al. (2014) would not require the contribution from a LEPP. Moreover, Cristallo et al. (2015a) found that the LEPP is not completely necessary to explain the solar composition of $s$-only isotopes when considering other aspects in their models such as rotation or star formation rate uncertainties. Indeed, our $[h s / l s]$ trend for thick disc stars resembles that of intermediate-mass AGB yields $\left(5-6 M_{\odot}\right)$ by Cristallo et al. (2015b), thus suggesting that the $s$-process production of our 
thick disc stars is dominated by intermediate-mass instead of low-mass AGB stars. On the contrary, this missing component of light $s$ elements might be explained by the contribution of massive stars which can reach up to $30 \%$ as shown in Pignatari et al. (2010). In a recent work, Bisterzo et al. (2017) reports that by including the contribution of fast-rotating metal-poor stars to the weak $s$-process (using the yields from Frischknecht et al. 2016) the solar abundances of Sr-Y-Zr can be increased, which could partially account for the solar LEPP. However, the inclusion of these yields has a major effect on the abundances of light- $s$ at very low metallicities, as previously reported by Cescutti et al. (2013). Nevertheless, Bisterzo et al. (2017) point to the necessity of a combination of different $r$ - and $s$-process to totally account for the solar and the metal-poor LEPP. The study of other populations in the Galaxy and the late major improvements in GCE models will help to understand this issue in the future.

\section{Summary}

In this work we present a detailed and homogeneous study of chemical abundances of $\mathrm{Cu}, \mathrm{Zn}, \mathrm{Sr}, \mathrm{Y}, \mathrm{Zr}, \mathrm{Ba}, \mathrm{Ce}, \mathrm{Nd}$, and $\mathrm{Eu}$ for the HARPS-GTO sample which complements our previous studies on Li, C, O, iron-peak, and $\alpha$ elements in a homogeneous way. One of the advantages of this high-quality volume-limited sample is that it allows the GCE to be studied at high metallicities since it contains a high number of metal-rich stars. The purpose of this work is to evaluate the production and evolution of neutron-capture elements in the different populations of the Galaxy: thin disc, thick disc, and homr stars. Our main discoveries and conclusions are the following:

- For the thin disc population we find mostly flat trends up to solar metallicity for $\mathrm{Zr}, \mathrm{Ce}$, and $\mathrm{Nd}$, while $\mathrm{Sr}, \mathrm{Y}$, and Ba show slightly rising abundances as $[\mathrm{Fe} / \mathrm{H}]$ increases with maximum values around solar metallicity. Then, the trend changes and the abundances decrease with higher metallicities, with $\mathrm{Zr}, \mathrm{Ba}$, and $\mathrm{Nd}$ showing steeper declines. On the contrary, $\mathrm{Cu}$ presents a flat trend up to solar metallicity and then continuously increases reaching a maximum $[\mathrm{Cu} / \mathrm{Fe}] \sim 0.3$ at $[\mathrm{Fe} / \mathrm{H}] \sim 0.4$ dex; instead, $\mathrm{Zn}$ remains practically flat at all $[\mathrm{Fe} / \mathrm{H}]$ and Eu shows a continuous lowering trend as $[\mathrm{Fe} / \mathrm{H}]$ increases. When comparing our observations of heavy-s and light $s$ elements with GCE models from Bisterzo et al. (2017) we observe that the general trends of thin disc stars are well reproduced, but our maximum abundances are delayed by $0.2-0.3$ dex with respect to the models. On the other hand, the $\mathrm{Cu}$ and $\mathrm{Zn}$ increases along $[\mathrm{Fe} / \mathrm{H}]$ for thin disc stars can be reproduced by the models of Romano et al. (2010) considering normal SNe II yields.

- Thick disc stars present disjunct abundances for $\mathrm{Zn}$ and $\mathrm{Eu}$, resembling the behaviour of $\alpha$ elements, although $\mathrm{Zn}$ remains mostly flat in our $[\mathrm{Fe} / \mathrm{H}]$ range. The elements $\mathrm{Eu}$ and $\mathrm{Zr}$ present steeper lowering trends of abundances towards higher metallicities, whereas $\mathrm{Cu}$ shows a steep increase as $[\mathrm{Fe} / \mathrm{H}]$ rises. The abundances of $\mathrm{Y}$ and $\mathrm{Ba}$ for thick disc stars are mainly subsolar; $\mathrm{Ce}$ and $\mathrm{Sr}$ present close to solar abundances on average; and $\mathrm{Zn}, \mathrm{Zr}, \mathrm{Nd}$, and Eu show super-solar abundances for most of the thick disc stars. The K-S tests reject the hypothesis that the abundances of thick disc stars and thin disc stars at $[\mathrm{Fe} / \mathrm{H}]<-0.2$ dex belong to the same parent population for $\mathrm{Zn}, \mathrm{Zr}, \mathrm{Ba}$, and $\mathrm{Eu}$. In general, none of the GCE models tested here can match the observations of thick stars except for $\mathrm{Cu}$. However, we note that the low number of thick disc stars at $[\mathrm{Fe} / \mathrm{H}]<-0.8$ dex in our sample might be producing different trends than expected.

- We find that the homr stars show clear different abundances of $\mathrm{Cu}, \mathrm{Zn}, \mathrm{Y}, \mathrm{Ba}, \mathrm{Nd}$, and $\mathrm{Eu}$ when compared to the thin disc stars, further supporting the different chemical enrichment of this population first discovered by (Adibekyan et al. 2011). The abundances of $\mathrm{Cu}, \mathrm{Zn}, \mathrm{Nd}$, and $\mathrm{Eu}$ are higher when compared to thin disc stars at the same $[\mathrm{Fe} / \mathrm{H}]$, whereas the abundances of $\mathrm{Ba}$ and $\mathrm{Y}$ are lower on average. We have performed K-S tests that confirm this behaviour; $\mathrm{Cu}$ and $\mathrm{Zn}$ show the highest distance between the two populations.

- By comparing the pure $r$ element Eu with the heavy-s elements we can see how at lower metallicities the earlier production of $r$-process elements by massive stars provides high $[\mathrm{Eu} / \mathrm{Ba}]$ and $[\mathrm{Eu} / \mathrm{Ce}]$ since $\mathrm{Ba}$ and $\mathrm{Ce}$ do not receive a major contribution of $r$-process in our metallicity range. On the other hand, the $[\mathrm{Eu} / \mathrm{Nd}]$ ratio is less steep because $50 \%$ of $\mathrm{Nd}$ is produced by the $r$-process. We also find that the $[\mathrm{Eu} / \mathrm{O}] \mathrm{ra}-$ tio is negative at lower metallicities and increases slightly to become flat at $[\mathrm{Fe} / \mathrm{H}] \sim-0$ dex. This trend shows that the masses of SNe progenitors producing $\mathrm{O}$ are higher than those producing Eu (Travaglio et al. 1999). Moreover, the steeper and more negative trends of $[\mathrm{Y} / \mathrm{O}]$ and $[\mathrm{Ba} / \mathrm{O}]$ agree with the production of these elements by AGB stars since they have longer lifetimes than the massive stars contributing to $\mathrm{Eu}$ and $\mathrm{O}$.

- We find that for the thin disc stars the ratio $[h s / l s]$ shows the expected behaviour of increasing values towards decreasing metallicities. This is a reflection of the higher neutron exposure as the metallicity declines (more neutrons per $\mathrm{Fe}$ seed), so for $[\mathrm{Fe} / \mathrm{H}] \gtrsim-0.5 \mathrm{dex}$ the $s$-process peaks at $\mathrm{Sr}-\mathrm{Y}-\mathrm{Zr}$, for intermediate metallicities the $s$-process mainly produces the second-peak (Ba-La-Ce-Sm-Nd) and for halo metallicities the high neutron exposure allows for the formation of $\mathrm{Pb}$, the heaviest $s$-process element. However, the thick disc population presents negative $[h s / l s]$ supporting previous findings of a missing contribution for light- $s$ elements at low metallicities (Travaglio et al. 2004a), which explains the higher than expected abundances of elements such as $\mathrm{Sr}$, $\mathrm{Y}$, and Zr. Nevertheless, we show that the low $[h s / l s]$ of our thick disc might be explained with yields of intermediatemass AGB stars (Cristallo et al. 2015b) instead of the major expected contribution from low-mass AGB stars. Finally, we also find an increase in light-s abundances with respect to heavy- $s$ at super-solar metallicities which might be produced by the contribution of metal-rich AGB stars (Karakas \& Lugaro 2016). Interestingly, homr stars mostly present positive $[h s / l s]$ ratios, whereas thin disc stars at simi$\operatorname{lar}[\mathrm{Fe} / \mathrm{H}]$ are spread at positive and negative ratios.

Our work demonstrates that even in the era of very large surveys, homogeneous and high-precision data of relatively small samples - even though this is probably the largest of its kind - can provide very important insights for our understanding of stellar nucleosynthesis and Galactic chemical evolution.

Acknowledgements. E.D.M., V.Zh.A., N.C.S., and S.G.S. acknowledge the support from Fundação para a Ciência e a Tecnologia (FCT) through national funds and from FEDER through COMPETE2020 by the following grants UID/FIS/04434/2013 \& POCI-01-0145-FEDER-007672, PTDC/FISAST/7073/2014 \& POCI-01-0145-FEDER-016880, and PTDC/FISAST/1526/2014 \& POCI-01-0145-FEDER-016886. E.D.M. acknowledges the support from the fellowship SFRH/BPD/76606/2011 funded by FCT (Portugal) and from the Investigador FCT contract IF/00849/2015/CP1273/CT0003. V.Zh.A., N.C.S., and S.G.S. also acknowledge the support from FCT through Investigador FCT contracts IF/00650/2015/CP1273/CT0001, IF/00169/2012/ 
CP0150/CT0002, and IF/00028/2014/CP1215/CT0002 funded by FCT (Portugal) and POPH/FSE (EC). J.I.G.H. acknowledges financial support from the Spanish Ministry of Economy and Competitiveness (MINECO) under the 2013 Ramón y Cajal program MINECO RYC-2013-14875, and the Spanish ministry project MINECO AYA2014-56359-P. We thank Sara Bisterzo and Donatella Romano for kindly providing their GCE models. This research has made use of the SIMBAD database operated at CDS, Strasbourg (France), the IRAF facility, and the VALD3 database. Finally, we are sincerely grateful to the anonymous referee, whose careful review helped to improve this paper.

\section{References}

Adibekyan, V. Z., Santos, N. C., Sousa, S. G., \& Israelian, G. 2011, A\&A, 535, L11

Adibekyan, V. Z., Sousa, S. G., Santos, N. C., et al. 2012, A\&A, 545, A32 Adibekyan, V. Z., Figueira, P., Santos, N. C., et al. 2013, A\&A, 554, A44 Adibekyan, V., Figueira, P., Santos, N. C., et al. 2015, A\&A, 583, A94

Allen, D. M., \& Porto de Mello, G. F. 2011, A\&A, 525, A63

Allende Prieto, C., Barklem, P. S., Lambert, D. L., \& Cunha, K. 2004, A\&A, 420, 183

Arlandini, C., Käppeler, F., Wisshak, K., et al. 1999, ApJ, 525, 886

Astraatmadja, T. L., \& Bailer-Jones, C. A. L. 2016, ApJ, 832, 137

Barbuy, B., Friaça, A. C. S., da Silveira, C. R., et al. 2015, A\&A, 580, A40

Battistini, C., \& Bensby, T. 2016, A\&A, 586, A49

Bensby, T., Feltzing, S., \& Lundström, I. 2003, A\&A, 410, 527

Bensby, T., Feltzing, S., \& Oey, M. S. 2014, A\&A, 562, A71

Bertran de Lis, S., Delgado Mena, E., Adibekyan, V. Z., Santos, N. C., \& Sousa, S. G. 2015, A\&A, 576, A89

Bessell, M. S., Castelli, F., \& Plez, B. 1998, A\&A, 333, 231

Bisterzo, S., Pompeia, L., Gallino, R., et al. 2005, Nucl. Phys. A, 758, 284

Bisterzo, S., Travaglio, C., Gallino, R., Wiescher, M., \& Käppeler, F. 2014, ApJ, 787, 10

Bisterzo, S., Travaglio, C., Wiescher, M., et al. 2016, J. Phys. Conf. Ser., 665 012023

Bisterzo, S., Travaglio, C., Wiescher, M., Käppeler, F., \& Gallino, R. 2017, ApJ, 835, 97

Bressan, A., Marigo, P., Girardi, L., et al. 2012, MNRAS, 427, 127

Burbidge, E. M., Burbidge, G. R., Fowler, W. A., \& Hoyle, F. 1957, Rev. Mod. Phys., 29, 547

Busso, M., Gallino, R., \& Wasserburg, G. J. 1999, ARA\&A, 37, 239

Castro, S., Porto de Mello, G. F., \& da Silva, L. 1999, MNRAS, 305, 693

Cayrel, R. 1988, in The Impact of Very High S/N Spectroscopy on Stellar Physics, eds. G. Cayrel de Strobel, \& M. Spite, IAU Symp., 132, 345

Cescutti, G., Chiappini, C., Hirschi, R., Meynet, G., \& Frischknecht, U. 2013 , A\&A, 553, A51

Chabrier, G. 2001, ApJ, 554, 1274

Cowan, J. J., \& Thielemann, F.-K. 2004, Physics Today, 57, 47

Cristallo, S., Abia, C., Straniero, O., \& Piersanti, L. 2015a, ApJ, 801, 53

Cristallo, S., Straniero, O., Piersanti, L., \& Gobrecht, D. 2015b, ApJS, 219, 40

da Silva, L., Girardi, L., Pasquini, L., et al. 2006, A\&A, 458, 609

Delgado Mena, E., Israelian, G., González Hernández, J. I., et al. 2014, A\&A, 562, A92

Delgado Mena, E., Bertrán de Lis, S., Adibekyan, V. Z., et al. 2015, A\&A, 576, A69

D’Orazi, V., Magrini, L., Randich, S., et al. 2009, ApJ, 693, L31

Duffau, S., Caffau, E., Sbordone, L., et al. 2017, A\&A, 604, A128

Fishlock, C. K., Karakas, A. I., Lugaro, M., \& Yong, D. 2014, ApJ, 797, 44

Flower, P. J. 1996, ApJ, 469, 355

Frischknecht, U., Hirschi, R., Pignatari, M., et al. 2016, MNRAS, 456, 1803

Gilmore, G., Randich, S., Asplund, M., et al. 2012, The Messenger, 147, 25

González Hernández, J. I., Israelian, G., Santos, N. C., et al. 2010, ApJ, 720, 1592

González Hernández, J. I., Delgado-Mena, E., Sousa, S. G., et al. 2013, A\&A, 552, A6

Grevesse, N., \& Sauval, A. J. 1998, Space Sci. Rev., 85, 161

Heijmans, J., Asplund, M., Barden, S., et al. 2012, in Ground-based and Airborne Instrumentation for Astronomy IV, Proc. SPIE, 8446, 84460W

Heiter, U., Lind, K., Asplund, M., et al. 2015, Phys. Scr., 90, 054010

Israelian, G., Bertran de Lis, S., Delgado Mena, E., \& Adibekyan, V. Z. 2014 Mem. Soc. Astron. Ital., 85, 265

Kappeler, F., Beer, H., \& Wisshak, K. 1989, Rept. Progr. Phys., 52, 945

Käppeler, F., Gallino, R., Bisterzo, S., \& Aoki, W. 2011, Rev. Mod. Phys., 83, 157
Karakas, A. I. 2016, Mem. Soc. Astron. Ital., 87, 229

Karakas, A. I., \& Lugaro, M. 2016, ApJ, 825, 26

Kobayashi, C., \& Nomoto, K. 2009, ApJ, 707, 1466

Kurucz, R. 1993, in ATLAS9 Stellar Atmosphere Programs and $2 \mathrm{~km} \mathrm{~s}^{-1}$ grid. Kurucz CD-ROM (Cambridge, Mass.: Smithsonian Astrophysical Observatory), 13

Lindegren, L., \& Feltzing, S. 2013, A\&A, 553, A94

Lo Curto, G., Mayor, M., Benz, W., et al. 2010, A\&A, 512, A48

Lodders, K., Palme, H., \& Gail, H.-P. 2009, in Landolt Börnstein, Vol. VI/4B, Chap. 4.4

Maiorca, E., Magrini, L., Busso, M., et al. 2012, ApJ, 747, 53

Matteucci, F., Raiteri, C. M., Busson, M., Gallino, R., \& Gratton, R. 1993, A\&A, 272,421

Mayor, M., Pepe, F., Queloz, D., et al. 2003, The Messenger, 114, 20

McWilliam, A., Matteucci, F., Ballero, S., et al. 2008, AJ, 136, 367

Mikolaitis, Š., de Laverny, P., Recio-Blanco, A., et al. 2017, A\&A, 600, A22

Mishenina, T. V., Kovtyukh, V. V., Soubiran, C., Travaglio, C., \& Busso, M. 2002, A\&A, 396, 189

Mishenina, T. V., Pignatari, M., Korotin, S. A., et al. 2013, A\&A, 552, A128

Mortier, A., Sousa, S. G., Adibekyan, V. Z., Brandão, I. M., \& Santos, N. C. 2014, A\&A, 572, A95

Pignatari, M., Gallino, R., Heil, M., et al. 2010, ApJ, 710, 1557

Pignatari, M., Hirschi, R., Wiescher, M., et al. 2013, ApJ, 762, 31

Pompéia, L., \& Allen, D. M. 2008, A\&A, 488, 723

Prantzos, N., Hashimoto, M., \& Nomoto, K. 1990, A\&A, 234, 211

Prochaska, J. X., Naumov, S. O., Carney, B. W., McWilliam, A., \& Wolfe, A. M. 2000, AJ, 120, 2513

Raiteri, C. M., Gallino, R., Busso, M., Neuberger, D., \& Kaeppeler, F. 1993, ApJ, 419, 207

Reddy, B. E., Tomkin, J., Lambert, D. L., \& Allende Prieto, C. 2003, MNRAS, 340, 304

Reddy, B. E., Lambert, D. L., \& Allende Prieto, C. 2006, MNRAS, 367, 1329

Romano, D., \& Matteucci, F. 2007, MNRAS, 378, L59

Romano, D., Karakas, A. I., Tosi, M., \& Matteucci, F. 2010, A\&A, 522, A32

Ryabchikova, T., Piskunov, N., Kurucz, R. L., et al. 2015, Phys. Scr, 90, 054005

Saito, Y.-J., Takada-Hidai, M., Honda, S., \& Takeda, Y. 2009, PASJ, 61, 549

Santos, N. C., Israelian, G., \& Mayor, M. 2004, A\&A, 415, 1153

Santos, N. C., Mayor, M., Bonfils, X., et al. 2011, A\&A, 526, A112

Sneden, C., Gratton, R. G., \& Crocker, D. A. 1991, A\&A, 246, 354

Sneden, C., Cowan, J. J., \& Gallino, R. 2008, ARA\&A, 46, 241

Sneden, C. A. 1973, Ph.D. Thesis, The University of Texas at Austin, USA

Sousa, S. G., Santos, N. C., Israelian, G., Mayor, M., \& Monteiro, M. J. P. F. G. 2007, A\&A, 469, 783

Sousa, S. G., Santos, N. C., Mayor, M., et al. 2008, A\&A, 487, 373

Sousa, S. G., Santos, N. C., Israelian, G., et al. 2011 a, A\&A, 526, A99

Sousa, S. G., Santos, N. C., Israelian, G., Mayor, M., \& Udry, S. 2011b, A\&A, 533, A141

Sousa, S. G., Santos, N. C., Adibekyan, V., Delgado-Mena, E., \& Israelian, G. 2015, A\&A, 577, A67

Spite, M., \& Spite, F. 1978, A\&A, 67, 23

Steinmetz, M. 2003, in Gaia Spectroscopy: Science and Technology, ed. U. Munari, ASP Conf. Ser., 298, 381

Suárez-Andrés, L., Israelian, G., González Hernández, J. I., et al. 2016, A\&A, 591, A69

Suárez-Andrés, L., Israelian, G., González Hernández, J. I., et al. 2017, A\&A, 599, A96

Torres, G. 2010, AJ, 140, 1158

Travaglio, C., Galli, D., Gallino, R., et al. 1999, ApJ, 521, 691

Travaglio, C., Gallino, R., Arnone, E., et al. 2004a, ApJ, 601, 864

Travaglio, C., Hillebrandt, W., Reinecke, M., \& Thielemann, F.-K. 2004b, A\&A, 425, 1029

Trippella, O., Busso, M., Maiorca, E., Käppeler, F., \& Palmerini, S. 2014, ApJ, 787, 41

Tsantaki, M., Sousa, S. G., Adibekyan, V. Z., et al. 2013, A\&A, 555, A150

van Leeuwen, F. 2007, A\&A, 474, 653

Wilson, J. C., Hearty, F., Skrutskie, M. F., et al. 2010, in Ground-based and Airborne Instrumentation for Astronomy III, Proc. SPIE, 7735, 77351C

Woosley, S. E., \& Weaver, T. A. 1995, ApJS, 101, 181

Yan, H. L., Shi, J. R., \& Zhao, G. 2015, ApJ, 802, 36

Yanny, B., Rockosi, C., Newberg, H. J., et al. 2009, AJ, 137, 4377

Zhao, G., Mashonkina, L., Yan, H. L., et al. 2016, ApJ, 833, 225 


\section{Appendix A: Additional figure}

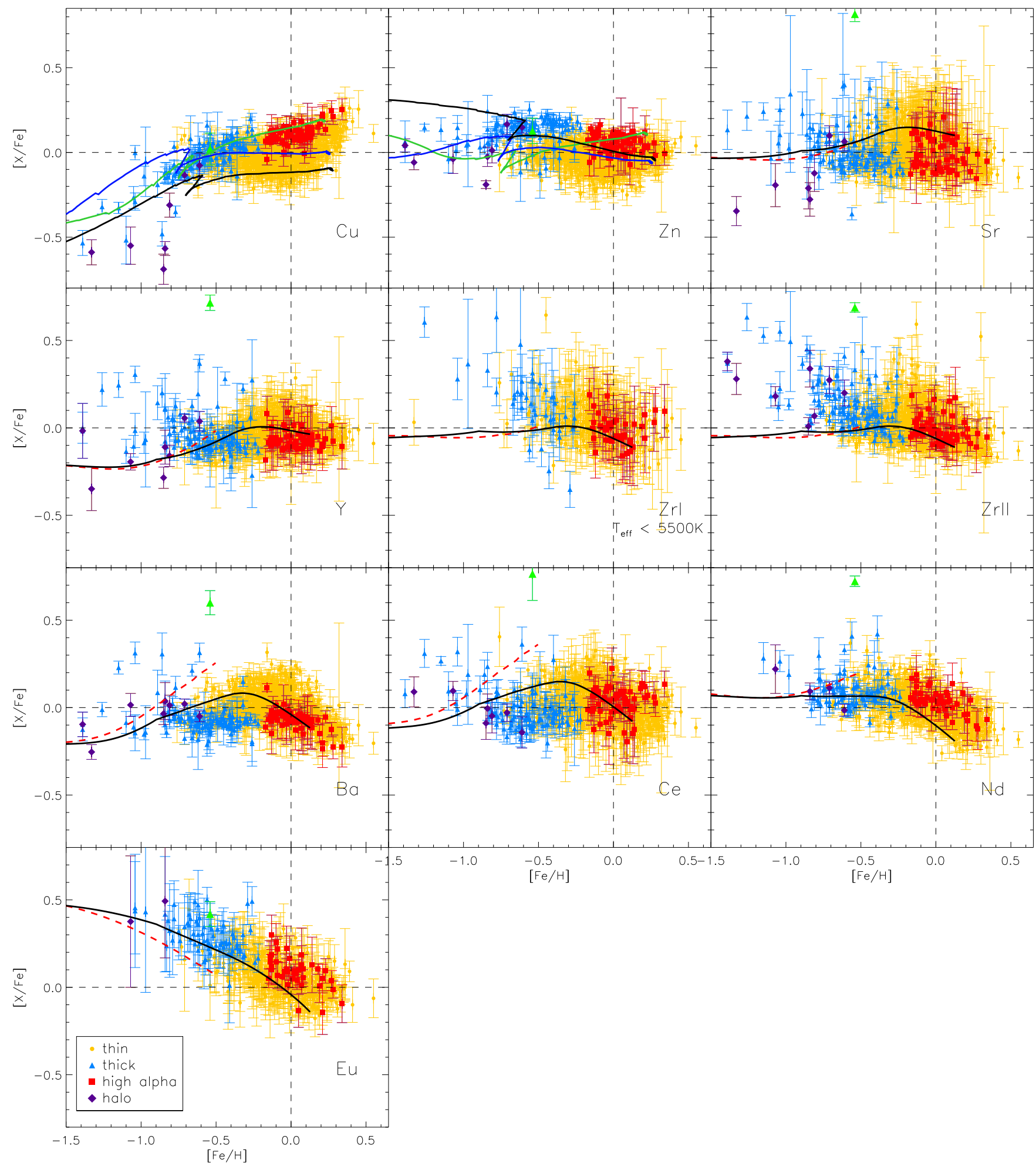

Fig. A.1. Final $[\mathrm{X} / \mathrm{Fe}]$ ratios as a function of $[\mathrm{Fe} / \mathrm{H}]$ for the full sample. The different stellar populations are depicted with different colours and symbols as explained in the legend. The green bigger triangle is the $s$-enriched star HD 11397. For $\mathrm{Cu}$ and $\mathrm{Zn}$ we overplot the GCE models 1 , 4, and 5 (green, blue, and black lines, respectively) from Romano et al. (2010). For the rest of the elements we show the GCE models from Bisterzo et al. (2017) for the thin disc (black lines) and the thick disc (red dashed lines). 